\title{
Speculative Attacks with Multiple Targets
}

\author{
Junichi Fujimoto* \\ University of Tokyo
}

First Version: October 2009

This Version: November 2011

\begin{abstract}
This paper examines a global games model of speculative attacks in which speculators can choose to attack any one of a number of targets. In the canonical global games model with a single target, it is well known that there exists a unique equilibrium that survives the iterative deletion of dominated strategies, characterized by the threshold values of the private signal and the fundamentals. This paper shows that with two targets, iterative deletion of dominated strategies yields a unique combination of threshold signal functions that are nondecreasing in the private signals of the other target's fundamentals, and threshold fundamentals functions that are increasing in the other target's fundamentals. The result is shown to extend to environments with any $N$ symmetric targets. The key argument is to combine the iterative deletion procedure with the contraction mapping theorem. The paper then goes through a number of numerical examples and shows, among other results, that more accurate private signals have a decoupling effect on the outcomes of attack on different countries. Finally, this paper introduces public information and shows that the sufficient condition for unique equilibrium threshold functions is very similar to that for a unique equilibrium in the single-target model.
\end{abstract}

KEYWORDS: Currency crises; Speculative attacks; Global games JEL CLASSIFICATION: F31, D84, D82

${ }^{*}$ I thank Andrew Atkeson, Toni Braun, Matthias Doepke, Gary Hansen, Christian Hellwig, Selahattin Imrohoroglu, Noritaka Kudoh, Junsang Lee, Daisuke Miyakawa, Stephen Morris, Guillaume Plantin, Jakub Steiner, and seminar participants at the 2009 GRIPS Conference of Economic Growth, Dynamics, and Policies, the 2010 Academica Sinica Conference on Growth, Trade and Dynamics, the 2010 Econometric Society World Congress, the 2010 European Economic Association Annual Congress, the Development Bank of Japan, and the University of Hokkaido for comments and suggestions. This research was supported by the Japan Securities Scholarship Foundation and the Zengin Foundation for Studies on Economics and Finance. E-mail: junichif@e.u-tokyo.ac.jp 


\section{Introduction}

Global games of regime change are "coordination games of incomplete information in which a status quo is abandoned once a sufficiently large fraction of agents attack it" (Angeletos, Hellwig, and Pavan (2007)). In a typical setup, agents receive a noisy private signal of the fundamentals, which represents the strength of the regime, or the status quo. The agents then individually decide whether or not to attack, or more generally take an action against, the regime, and when the fraction of agents attacking exceeds a certain threshold that depends on the fundamentals, the attack succeeds and the regime is abandoned. Since the seminal work of Morris and Shin (1998), which applied such games to analyze speculative attacks against a currency peg, these games have been actively applied to model a wide range of crisis situations.

This paper extends the literature on global games of regime change by allowing for multiple regimes, or targets, that agents can choose to attack. Such a situation may arise in a number of real-life environments. In the context of currency crises, speculators may face multiple emerging countries with currency pegs, and choose from among these currencies to allocate their limited resources for attack, such as wealth and informational capacity. For example, during the Asian crisis of 1997-98, there were a number of Asian currencies largely pegged to the U.S. dollar that came under speculative attack. Similar scenarios apply to sovereign debt crises, including the recent European sovereign debt crisis, in which the debt and credit default swaps of several countries, rumored to be facing potential default, have been subject to speculative trading. Other possible applications include a situation in which investors decide which project to invest in, or rioters choose which government facility to attack, where the success of investment or attack requires participation of at least a certain mass of agents. ${ }^{1}$ So, while this paper places the discussion in the context of currency crises, its implications extend to a much broader context.

In the canonical global games model with a single target, it is well known (see, e.g., Morris and Shin (1998)) that iterative deletion of (strictly) dominated strategies yields a unique equilibrium ${ }^{2}$ characterized by a threshold value of the private signal that determines whether an agent participates in an attack, and by a threshold value of the fundamentals that determines whether the regime is abandoned. This paper shows that with two targets, with potentially different precision of signals and cost of attack, iterative

\footnotetext{
${ }^{1}$ The global games approach is used to analyze investment in projects in Dasgupta (2007), and political riots in Atkeson (2000), although in an environment with a single project or target of attack.

${ }^{2}$ Multiple equilibria may arise, however, when there is public information of the fundamentals, either exogenous (Hellwig (2002)) or endogenous (Angeletos and Werning (2006), Hellwig, Mukherji, and Tsyvinski (2006)) that is sufficiently precise.
} 
deletion of strictly dominated strategies results in a unique combination of equilibrium threshold signal functions and threshold fundamentals functions. As a consequence, the equilibrium is unique up to ties, and the outcomes of attack are uniquely determined given the fundamentals. The present paper then shows that the result extends to environments with any $N$ symmetric countries.

The basic argument behind these results is as follows. By extending the iterative deletion procedure to the multiple target environment, one can define an operator on an adequate space of functions. The existence and uniqueness of the equilibrium threshold functions then follows by showing that this operator is a contraction, and by then invoking the contraction mapping theorem. The resulting threshold signal functions are nondecreasing in the private signals of other targets' fundamentals, whereas threshold fundamentals functions are increasing ${ }^{3}$ in the fundamentals of other targets.

While the equilibrium threshold functions cannot be obtained in closed form, they can be computed through a relatively simple numerical algorithm, thanks to the contraction property. In order to explore further properties of these functions and the associated outcomes of attack, this paper explores, mainly focusing on the two-country case, several numerical examples, and obtains several interesting results. First, with multiple targets, a target's sustainability depends crucially on the fundamentals of the other target, and not simply on its own fundamentals; a target's fundamentals are evaluated relative to those of the other target. Second, the presence of a second target makes the survival of a target more likely, if the total measure of speculators is fixed; if the measure of speculators doubles as does the number of targets, however, the region of fundamentals in which both countries sustain the peg becomes smaller than when the two countries separately face the speculative attack. Third, an increased precision of speculators' private signals has a decoupling effect; since more accurate signals allow speculators to better discern the country with the weaker fundamentals and to concentrate their attack on it, two countries are more likely to face different outcomes of attack.

Finally, this paper examines an extension of introducing exogenous public information. The sufficient condition for unique equilibrium threshold functions, which guarantees that the relevant operator is a contraction, is expressed in terms of the relative precision of public and private information, and closely resembles the known sufficient condition for equilibrium uniqueness in the standard model with a single target. In view of this finding, this paper relates its contraction argument to the standard proof of equilibrium uniqueness through iterative deletion of dominated strategies.

This paper is related to studies that explore games of incomplete information with mul-

\footnotetext{
${ }^{3}$ Throughout this paper, increasing (decreasing) implies strictly increasing (decreasing), and nondecreasing (nonincreasing) implies weakly increasing (decreasing).
} 
tidimensional actions, types or payoff parameters. McAdams (2003) provides a sufficient condition for the existence of an isotone pure strategy equilibrium in a class of games of incomplete information with multidimensional actions and types. Oury (2005) considers global games with multidimensional actions and payoff parameters, and shows that the limit uniqueness result of Frankel, Morris, and Pauzner (2003) extends to this setting. In both these studies, the elements of multidimensional actions can be chosen independently. In contrast, in the present paper, speculators can attack at most one target, or more generally, can sell at most a fixed amount of all target currencies combined. This assumption imposes restrictions on the possible combinations of actions toward each country, and as an important consequence, there is no complementarity within own actions, unlike Oury (2005).

This paper also has some similarities with Steiner (2007), which examines a mobile game in which agents in a sector coordinate to make an investment and have the outside option of leaving the current coordination problem (i.e., sector) for other problems (i.e., other sectors). However, while agents in the present paper receive private signals of multiple fundamentals, in Steiner (2007) agents receive private signals only of the fundamentals of the current sector and, consequently, the equilibrium remains characterized by threshold values, instead of threshold functions.

Finally, there are a number of studies that adopt the contraction mapping approach to examine the equilibrium of games with strategic complementarities. Levin (2001) explores an overlapping generations games in which agents, having an incentive to coordinate and facing uncertainty in future states of the world, sequentially choose their actions. Using the contraction mapping theorem, Levin (2001) shows the existence of a unique equilibrium when agents believe their actions will have no influence on future play. Mason and Valentinyi (2007) applies the contraction mapping argument to prove the existence and uniqueness of equilibrium in a class of games of incomplete information that exhibits, unlike the typical global games environment, large heterogeneity in agents' types. Mathevet (2010) considers finite global games in which the existence of pure strategy equilibria follows from the standard argument for supermodular games ${ }^{4}$, and establishes uniqueness of equilibrium by showing that the best response function is weak contraction. The present paper considers a multidimensional environment substantially different from these studies, and applies the contraction approach to the threshold fundamentals functions, instead of the best response function.

\footnotetext{
${ }^{4}$ For detailed discussions on supermodular games, see e.g., Milgrom and Roberts (1990) and Vives (1990).
} 


\section{Model}

\subsection{Basic Environment}

The model follows a simplified version of that in Morris and Shin (1998), except that speculators can now choose to attack one of multiple potential targets, creating a new dimension of coordination. There are $N>1$ countries indexed by $j \in\{1,2, \ldots, N\}$, whose currencies are also referred to as currency $j$. Each currency is pegged to a foreign currency. Country $j$ 's economic fundamentals are denoted as $\theta^{j}$, and $\theta^{1}, \theta^{2}, \ldots, \theta^{N}$ are independently drawn from a uniform distribution over the entire real line.

There is a continuum of risk-neutral speculators, indexed by $i \in[0,1]$. Instead of observing the true values of $\theta^{j}$, speculators receive noisy private signals of them. Speculator $i$ 's private signal of $\theta^{j}$, denoted as $x_{i}^{j}$, is expressed as

$$
x_{i}^{j}=\theta^{j}+\epsilon_{i}^{j},
$$

where $\epsilon_{i}^{1}, \epsilon_{i}^{2}, \ldots, \epsilon_{i}^{N}$ are independently drawn from $N\left(0,1 / \beta^{j}\right), \beta^{j}>0 .{ }^{5}$

Based on these $N$ signals, speculators individually decide whether to attack any, but at most one, of the $N$ currencies. In what follows, a superscript $-j$ implies that the variable pertains to $N-1$ countries (or currencies) other than $j$. Speculator $i$ 's pure strategy is $\left(a_{i}^{1}, a_{i}^{2}, \ldots, a_{i}^{N}\right): \mathbb{R}^{N} \rightarrow\{0,1\}^{N}, \Sigma_{j=1}^{N} a_{i}^{j}\left(x_{i}^{j}, x_{i}^{-j}\right)=\{0,1\}$, where $a_{i}^{j}=1$ implies attacking currency $j$ and $a_{i}^{j}=0$ implies not attacking it. ${ }^{6}$ While I focus here on pure strategy, and also, do not consider partial attacks against a single or multiple currencies, the analysis below shows that it is without loss of generality, except for measure zero of marginal speculators facing equally attractive targets.

Denote by $A^{j}: \mathbb{R}^{N} \rightarrow[0,1]$ the measure, or the fraction since the measure of speculators equals one, of speculators attacking currency $j$, as a function of fundamentals $\theta \equiv\left(\theta^{1}, \theta^{2}, \ldots, \theta^{N}\right)=\left(\theta^{j}, \theta^{-j}\right)$. If the measure of speculators attacking $j$ equals or exceeds the realized value of the fundamentals $\theta^{j}$ or, equivalently, if $A^{j}\left(\theta^{j}, \theta^{-j}\right) \geq \theta^{j}$, the attack against $j$ is successful, forcing country $j$ to abandon the peg; currency $j$ then floats and depreciates, providing profits to those who attacked the currency. Otherwise, the

\footnotetext{
${ }^{5}$ The assumption that $\epsilon_{i}^{j}$ is normally distributed is stronger than is necessary for the results in Section 3 ; the argument goes through if $\epsilon_{i}^{j}$ is distributed according to a continuous probability density function (pdf) with support on the real line. The normality assumption is necessary, however, when allowing for public information in Section 5.1. The case of correlated signals is discussed in Section 5.2.

${ }^{6}$ Alternatively, I could rule out partial attacks (i.e., $\left.a_{i}^{j} \in(0,1)\right)$ from the outset and define speculator $i$ 's pure strategy as $a_{i}: \mathbb{R}^{N} \rightarrow\{0,1, \ldots, N\}$, where $a_{i}=j \in\{1,2, \ldots, N\}$ implies attacking country $j$, and $a_{i}=0$ implies attacking none. The current formulation, however, allows us to see that the main implications of the paper do not hinge on the exclusion of partial attacks.
} 
attack against currency $j$ is unsuccessful and $j$ sustains the peg. Thus, currency $j$ always abandons the peg for $\theta^{j} \leq 0$, and never does so for $\theta^{j}>1$. Since a speculator can attack at most one currency, $\Sigma_{j=1}^{N} A^{j}\left(\theta^{j}, \theta^{-j}\right) \leq 1$.

Speculators' payoffs are summarized in Table 1. The payoff for speculators who attack a particular currency depends only on the success of that attack, and not on the outcomes of attack for other currencies. Speculators attacking currency $j$ receive $1-c^{j}>0$ if $j$ abandons the peg, and $-c^{j}<0$ otherwise, where $c^{j} \in(0,1)$ is a transaction cost, which may differ across $j$. Speculators attacking none of the currencies receive 0 regardless of the outcome of the attack.

\begin{tabular}{lll}
\hline \hline & Currency $j$ abandons the peg & Currency $j$ sustains the peg \\
\hline Attack currency $j$ & $1-c^{j}$ & $-c^{j}$ \\
Attack no currency & 0 & 0 \\
\hline
\end{tabular}

Table 1: Payoffs.

\subsection{Two Benchmark Models}

Before starting the main analysis, I discuss below two related models with a similar environment, which will be used for comparison with the model of this paper.

\subsubsection{Multiple Targets with Public Information}

The first benchmark is a version of the model in which there are $N>1$ targets but where the fundamentals $\theta^{j}, j=\{1,2, \ldots, N\}$, are publicly observed. For simplicity, suppose that $c^{j}$ is the same for all countries. ${ }^{7}$ This can be considered a multi-country extension of Obstfeld (1996), which exhibits a tripartite division of fundamentals $\theta$ according to the resulting forms of equilibrium: All speculators attack and the peg is abandoned if $\theta \leq 0$, no speculator attacks and the peg is sustained if $\theta>1$, and both of these become equilibria if $\theta \in(0,1]$.

With multiple targets, the Nash equilibrium is described as follows. If $\theta^{j}>1$ for all $j$, no speculator attacks any currency and the peg is sustained for all $j$. If $\theta^{j} \leq 0$ for $j=\tilde{j}$ and $\theta^{j}>1$ for all $j \neq \tilde{j}$, all speculators attack currency $\tilde{j}$, and the peg is abandoned for $\tilde{j}$ and is sustained for all $j \neq \tilde{j}$. In all other cases, there will be multiple equilibria. If $\theta^{j} \leq 0$ for at least one $j$ and $\theta^{j} \leq 1$ for at least one other $j$, each speculator attacks one of

\footnotetext{
${ }^{7}$ When $c^{j}$ is allowed to vary with $j$, the analysis requires examining various cases according to the values of $c^{j}$ and $\theta^{j}$. While not difficult, such analysis does not provide additional insight on the difference between public and private information environments.
} 
the currencies with $\theta^{j} \leq 1$, and currencies attacked by any speculator abandon the peg. Finally, if $\theta^{j}>0$ for all $j$ and $\theta^{j} \leq 1$ for at least one $j$, either (1) each speculator attacks one of the currencies with $\theta^{j} \leq 1$, and currencies attacked by any speculator abandon the peg, or (2) no speculator attacks any currency and the peg is sustained for all $j$.

Therefore, as in Obstfeld (1996), the total fraction of speculators participating in an attack, $\sum_{j=1}^{N} A^{j}\left(\theta^{j}, \theta^{-j}\right)$, always equals 0 or 1 , and there may be a unique equilibrium or multiple equilibria, depending on the realization of fundamentals. The presence of multiple targets naturally complicates the structure of the equilibrium, but the economic outcomes for each country are very similar to the single-country case: The peg is abandoned for sure if $\theta^{j} \leq 0$, sustained for sure if $\theta^{j}>1$, and either outcome is possible if $\theta^{j} \in(0,1]$. The only additional restriction is that $\max \left[\theta^{j}, 0\right]$, summed over countries that abandon the peg, cannot exceed 1, which is the largest possible fraction of attackers. So, whether a country sustains the peg depends little on the fundamentals of other countries, which contrasts with the private information case analyzed in this paper.

\subsubsection{Single Target with Private Information}

The second benchmark is the canonical global games model with a single target. Since $N=1$, the superscript $j$ denoting country $j$ is redundant, and hence is removed; otherwise, all assumptions in Section 2.1 are sustained. As is well known, in this environment, there exists a unique Bayesian Nash equilibrium that survives the iterative deletion of dominated strategies. I briefly describe this iterative deletion procedure ${ }^{8}$, so as to facilitate the understanding of the corresponding procedure in the multiple target environment.

By assumption, the peg collapses for $\theta \leq 0=\underline{\theta}_{1}^{*}$ even if no speculator attacks the peg. Thus, given the signal $x_{i}$, the expected payoff from attacking is at least

$$
(1-c) \cdot \operatorname{Pr}\left(\theta \leq \underline{\theta}_{1}^{*} \mid x_{i}\right)-c \cdot\left(1-\operatorname{Pr}\left(\theta \leq \underline{\theta}_{1}^{*} \mid x_{i}\right)\right)=\operatorname{Pr}\left(\theta \leq \underline{\theta}_{1}^{*} \mid x_{i}\right)-c
$$

where $\operatorname{Pr}\left(\theta \leq \underline{\theta}_{1}^{*} \mid x_{i}\right)$ denotes the probability that $\theta \leq \underline{\theta}_{1}^{*}$, conditional on receiving $x_{i}$. Let $\Phi$ be the cumulative density function of the standard normal distribution. Since $\theta$ follows $N\left(x_{i}, 1 / \beta\right)$ given $x_{i}, \operatorname{Pr}\left(\theta \leq \underline{\theta}_{1}^{*} \mid x_{i}\right)=\Phi\left(\sqrt{\beta}\left(\underline{\theta}_{1}^{*}-x_{i}\right)\right)$, which is decreasing in $x_{i}$. Thus, not attacking is a dominated strategy for speculators with signals $x_{i}<\underline{x}_{1}^{*}$, where

$$
c=\operatorname{Pr}\left(\theta \leq \underline{\theta}_{1}^{*} \mid \underline{x}_{1}^{*}\right)=\Phi\left(\sqrt{\beta}\left(\underline{\theta}_{1}^{*}-\underline{x}_{1}^{*}\right)\right),
$$

which completes the first round of deletion.

\footnotetext{
${ }^{8}$ For a more detailed discussion on the iterative deletion of dominated strategies in this context, see e.g., Atkeson (2000).
} 
In the second round of deletion, it is taken as given that speculators with signals $x_{i} \leq \underline{x}_{1}^{*}$ will attack the currency. ${ }^{9} \quad$ Let $\operatorname{Pr}\left(x_{i} \leq \underline{x}_{1}^{*} \mid \theta\right)$ be the probability that $x_{i} \leq \underline{x}_{1}^{*}$, conditional on a given realization of $\theta$; from the law of large numbers, this is also the fraction of speculators with such signals. Since $x_{i}$ follows $N(\theta, 1 / \beta)$ conditional on $\theta$, $\operatorname{Pr}\left(x_{i} \leq \underline{x}_{1}^{*} \mid \theta\right)=\Phi\left(\sqrt{\beta}\left(\underline{x}_{1}^{*}-\theta\right)\right)$, which is decreasing in $\theta$. So, even under the most pessimistic belief about the success of attack, speculators must expect the peg to collapse for $\theta \leq \underline{\theta}_{2}^{*}$, where

$$
\underline{\theta}_{2}^{*}=\operatorname{Pr}\left(x_{i} \leq \underline{x}_{1}^{*} \mid \underline{\theta}_{2}^{*}\right)=\Phi\left(\sqrt{\beta}\left(\underline{x}_{1}^{*}-\underline{\theta}_{2}^{*}\right)\right) .
$$

Thus, not attacking is a dominated strategy for speculators with signals $x_{i}<\underline{x}_{2}^{*}$, where

$$
c=\operatorname{Pr}\left(\theta \leq \underline{\theta}_{2}^{*} \mid \underline{x}_{2}^{*}\right)=\Phi\left(\sqrt{\beta}\left(\underline{\theta}_{2}^{*}-\underline{x}_{2}^{*}\right)\right) .
$$

Clearly $\underline{\theta}_{2}^{*}>\underline{\theta}_{1}^{*}=0$, which implies $\underline{x}_{2}^{*}>\underline{x}_{1}^{*}$. This completes the second round of deletion.

Iterating this process, one obtains an increasing sequence $\left\{\underline{\theta}_{n}^{*}, \underline{x}_{n}^{*}\right\}_{n=1}^{\infty}$ such that in the $n$-th round of deletion, even under the most pessimistic belief about the success of an attack, speculators must expect the peg to collapse for $\theta \leq \underline{\theta}_{n}^{*}$, and not attacking is a dominated strategy for $x_{i}<\underline{x}_{n}^{*}$. Proceeding similarly from $\bar{\theta}_{1}=1$, one obtains a decreasing sequence $\left\{\bar{\theta}_{n}^{*}, \bar{x}_{n}^{*}\right\}_{n=1}^{\infty}$ such that in the $n$-th round of deletion, even under the most optimistic belief about the success of an attack, speculators must expect the peg to be sustained for $\theta>\bar{\theta}_{n}^{*}$, and attacking is a dominated strategy for $x_{i}>\bar{x}_{n}^{*}$.

The limits of these sequences, $\left\{\underline{\theta}_{\infty}^{*}, \underline{x}_{\infty}^{*}\right\}$ and $\left\{\bar{\theta}_{\infty}^{*}, \bar{x}_{\infty}^{*}\right\}$, must both be solutions to

$$
\begin{gathered}
c=\operatorname{Pr}\left(\theta \leq \theta_{s}^{*} \mid x_{s}^{*}\right)=\Phi\left(\sqrt{\beta}\left(\theta_{s}^{*}-x_{s}^{*}\right)\right), \\
\theta_{s}^{*}=\operatorname{Pr}\left(x_{i} \leq x_{s}^{*} \mid \theta_{s}^{*}\right)=\Phi\left(\sqrt{\beta}\left(x_{s}^{*}-\theta_{s}^{*}\right)\right) .
\end{gathered}
$$

But given $\Phi(a)=1-\Phi(-a),(6)$ and $(7)$ yield

$$
\begin{aligned}
& \theta_{s}^{*}=1-c \\
& x_{s}^{*}=\theta_{s}^{*}+\frac{1}{\sqrt{\beta}} \Phi^{-1}\left(\theta_{s}^{*}\right)=1-c+\frac{1}{\sqrt{\beta}} \Phi^{-1}(1-c),
\end{aligned}
$$

hence $\underline{\theta}_{\infty}^{*}=\bar{\theta}_{\infty}^{*}=\theta_{s}^{*}$ and $\underline{x}_{\infty}^{*}=\bar{x}_{\infty}^{*}=x_{s}^{*}$. Thus, with a single target, the equilibrium is characterized by a threshold signal $x_{s}^{*}$ and threshold fundamentals $\theta_{s}^{*}$ such that each speculator attacks if and only if $x_{i} \leq x_{s}^{*}$, and the peg is abandoned if and only if $\theta \leq \theta_{s}^{*}$.

\footnotetext{
${ }^{9}$ Having a weak, instead of strict, inequality here presumes that speculators attack when they are indifferent; such a tie-breaking rule is immaterial.
} 


\section{Analysis of the Main Model}

\subsection{Overview}

I now turn to the main model of this paper, which features multiple targets and private information. I show below that with $N=2$ countries, or with any $N$ symmetric countries, the equilibrium of this speculative attack game can be obtained by iterative deletion of dominated strategies. Such equilibrium takes the following form: speculator $i$ attacks currency $j$ if $x_{i}^{j}<x^{j *}\left(x_{i}^{-j}\right)$, and only if $x_{i}^{j} \leq x^{j *}\left(x_{i}^{-j}\right)$, where $x^{j *}: \mathbb{R}^{N-1} \rightarrow$ $\mathbb{R}$ is continuous and nondecreasing. The associated outcomes of attack are such that currency $j$ abandons the peg if and only if $\theta^{j} \leq \theta^{j *}\left(\theta^{-j}\right)$, where $\theta^{j *}: \mathbb{R}^{N-1} \rightarrow(0,1)$ is continuous and increasing. Thus, the equilibrium is characterized by a unique combination of threshold signal functions $x^{j *}$ and threshold fundamentals functions $\theta^{j *}$. As a consequence, the outcomes of attack for all countries are uniquely pinned down given the fundamentals, which contrasts with the public information case discussed above.

The argument proceeds as follows. First, I adapt the iterative deletion procedure discussed above to this multiple target environment. This procedure generates, for each country, an increasing sequence of threshold functions starting from 'below', and a decreasing sequence of threshold functions starting from 'above'. Second, I define an operator associated with these sequences, show that the operator is a contraction, and invoke the contraction mapping theorem to show that there is a unique fixed point of this operator, corresponding to the limits of the sequences of functions from iterative deletion. Finally, I show that the 'lower' and 'upper' limiting functions indeed coincide.

\subsection{Iterative Deletion of Dominated Strategies}

With multiple targets, speculators face not only the choice between attacking and not attacking, but also the choice of which country to attack, which complicates the procedure of iterative deletion of dominated strategies. To obtain the sequences of functions that converge to the equilibrium functions, one must combine deletion from 'above' and 'below', instead of proceeding separately as in the single-country case discussed in Section 2.2.2.

For any $\theta^{-j}$, let $\underline{\theta}_{n}^{j *}\left(\theta^{-j}\right)$ and $\bar{\theta}_{n}^{j *}\left(\theta^{-j}\right)$ denote, respectively, the value of $\theta^{j}$ associated with the most pessimistic and the most optimistic beliefs about the success of attack against currency $j$, at the beginning of the $n$-th round of deletion. For any $N$ signals $x_{i}=$ $\left(x_{i}^{1}, x_{i}^{2}, \ldots, x_{i}^{N}\right)=\left(x_{i}^{j}, x_{i}^{-j}\right)$, let $\underline{\Gamma}_{n}^{j *}\left(x_{i}\right)$ and $\bar{\Gamma}_{n}^{j *}\left(x_{i}\right)$ denote, respectively, the expected payoff from attacking currency $j$ under the belief that $j$ abandons the peg if and only if $\theta^{j} \leq \underline{\theta}_{n}^{j *}\left(\theta^{-j}\right)$, and that under the belief that $j$ abandons the peg if and only if $\theta^{j} \leq$ $\bar{\theta}_{n}^{j *}\left(\theta^{-j}\right)$. Associated with these extreme beliefs are functions $\underline{x}_{n}^{j *}$ and $\bar{x}_{n}^{j *}$ such that in 
the $n$-th round, not attacking $j$ is strictly dominated for $x_{i}^{j}<\underline{x}_{n}^{j *}\left(x_{i}^{-j}\right)$, and attacking $j$ is strictly dominated for $x_{i}^{j}>\bar{x}_{n}^{j *}\left(x_{i}^{-j}\right)$. Clearly, $\bar{x}_{n}^{j *}\left(x_{i}^{-j}\right) \geq \underline{x}_{n}^{j *}\left(x_{i}^{-j}\right)$.

Recall that for $\theta^{j} \leq 0$, currency $j$ abandons the peg even if no speculator attacks $j$, and for $\theta^{j}>1, j$ sustains the peg even if all speculators attack it. Thus, in the first round of deletion, speculators must believe, even under the most pessimistic belief about the success of attack against currency $j$, that $j$ abandons the peg for $\theta^{j} \leq 0=\underline{\theta}_{1}^{j *}\left(\theta^{-j}\right)$, and even under the most optimistic belief about the success of attack against $j$, that $j$ sustains the peg for $\theta^{j}>1=\bar{\theta}_{1}^{j *}\left(\theta^{-j}\right)$. Let us now compute $\underline{\Gamma}_{1}^{j *}$ and $\bar{\Gamma}_{1}^{j *}$. Since $\theta^{j}=x_{i}^{j}-\epsilon_{i}^{j}$,

$$
\operatorname{Pr}\left(\theta^{j} \leq \theta^{j *} \mid x_{i}^{j}\right)=\operatorname{Pr}\left(-\epsilon_{i}^{j} \leq \theta^{j *}-x_{i}^{j}\right)=\Phi\left(\sqrt{\beta^{j}}\left(\theta^{j *}-x_{i}^{j}\right)\right)
$$

for a fixed $\theta^{j *}$. Therefore, noting that $\theta^{-j}=x_{i}^{-j}-\epsilon_{i}^{-j}$,

$$
\begin{aligned}
\underline{\Gamma}_{1}^{j *}\left(x_{i}\right) & =\operatorname{Pr}\left(\theta^{j} \leq \underline{\theta}_{1}^{j *}\left(\theta^{-j}\right) \mid x_{i}^{j}, x_{i}^{-j}\right)-c^{j} \\
& =\int_{\epsilon_{i}^{-j} \in \mathbb{R}^{N-1}} \tilde{\phi}^{-j}\left(\epsilon_{i}^{-j}\right) \Phi\left(\sqrt{\beta^{j}}\left(\underline{\theta}_{1}^{j *}\left(x_{i}^{-j}-\epsilon_{i}^{-j}\right)-x_{i}^{j}\right)\right) d \epsilon_{i}^{-j}-c^{j},
\end{aligned}
$$

where $\tilde{\phi}^{-j}$ is the joint pdf of $N-1$ independent normal noises, $\epsilon_{i}^{-j}$. Letting $\phi$ be the pdf of the standard normal distribution, $\tilde{\phi}^{-j}\left(\epsilon_{i}^{-j}\right)=\Pi_{k \neq j} \sqrt{\beta^{k}} \phi\left(\sqrt{\beta^{k}} \epsilon_{i}^{k}\right)$. Similarly,

$$
\begin{aligned}
\bar{\Gamma}_{1}^{j *}\left(x_{i}\right) & =\operatorname{Pr}\left(\theta^{j} \leq \bar{\theta}_{1}^{j *}\left(\theta^{-j}\right) \mid x_{i}^{j}, x_{i}^{-j}\right)-c^{j} \\
& =\int_{\epsilon_{i}^{-j} \in \mathbb{R}^{N-1}} \tilde{\phi}^{-j}\left(\epsilon_{i}^{-j}\right) \Phi\left(\sqrt{\beta^{j}}\left(\bar{\theta}_{1}^{j *}\left(x_{i}^{-j}-\epsilon_{i}^{-j}\right)-x_{i}^{j}\right)\right) d \epsilon_{i}^{-j}-c^{j} .
\end{aligned}
$$

For any $x_{i}^{-j} \in \mathbb{R}^{N-1}, \underline{x}_{1}^{j *}\left(x_{i}^{-j}\right)$ is the value of $x_{i}^{j}$ such that not attacking currency $j$ is dominated for all $x_{i}^{j}<\underline{x}_{1}^{j *}\left(x_{i}^{-j}\right)$. Formally,

$$
\underline{x}_{1}^{j *}\left(x_{i}^{-j}\right)=\inf \left\{x_{i}^{j} \mid \underline{\Gamma}_{1}^{j *}\left(x_{i}\right) \leq \max \left\{0,\left(\bar{\Gamma}_{1}^{k *}\left(x_{i}\right)\right)_{k \neq j}\right\}\right\},
$$

where $\underline{x}_{1}^{j *}\left(x_{i}^{-j}\right)=-\infty$ if the infimum does not exist. ${ }^{10}$ Thus, for speculators with signals $x_{i}^{j}<\underline{x}_{1}^{j *}\left(x_{i}^{-j}\right)$, even under the most pessimistic belief about the success of attack against currency $j$ and the most optimistic beliefs about the success of attacks against other currencies, the expected payoff from attacking $j$ exceeds that from other alternatives (i.e., attacking other currencies or attacking none). Similarly, $\bar{x}_{1}^{j *}\left(x_{i}^{-j}\right)$ is defined as

$$
\bar{x}_{1}^{j *}\left(x_{i}^{-j}\right)=\sup \left\{x_{i}^{j} \mid \bar{\Gamma}_{1}^{j *}\left(x_{i}\right) \geq \max \left\{0,\left(\underline{\Gamma}_{1}^{k *}\left(x_{i}\right)\right)_{k \neq j}\right\}\right\},
$$

\footnotetext{
${ }^{10}$ The infimum may not exist when $c^{j}>c^{k}$, since for sufficiently small $x_{i}^{k}, \bar{\Gamma}_{1}^{k *}\left(x_{i}\right)=\Phi\left(1-x_{i}^{k}\right)-c^{k}>$ $1-c^{j}=\lim _{x_{i}^{j} \rightarrow-\infty} \underline{\Gamma}_{1}^{j *}\left(x_{i}\right)$, hence $\underline{\Gamma}_{1}^{j *}\left(x_{i}\right)<\bar{\Gamma}_{1}^{k *}\left(x_{i}\right)$ for arbitrarily small $x_{i}^{j}$. Similar situations may arise in subsequent rounds of deletion.
} 
where $\bar{x}_{1}^{j *}\left(x_{i}^{-j}\right)=-\infty$ if the supremum does not exist. ${ }^{11}$ Given $x_{i}^{-j}, \bar{x}_{1}^{j *}\left(x_{i}^{-j}\right)$ is the value of $x_{i}^{j}$ such that for $x_{i}^{j}>\bar{x}_{1}^{j *}\left(x_{i}^{-j}\right)$, even under the most optimistic belief about the success of attack against currency $j$ and the most pessimistic beliefs about the success of attacks against other currencies, speculators prefer not to attack $j$.

In the second round, it is taken as given that speculators with signals $x_{i}^{j}<\underline{x}_{1}^{j *}\left(x_{i}^{-j}\right)$ will attack currency $j$, and those with signals $x_{i}^{j}>\bar{x}_{1}^{j *}\left(x_{i}^{-j}\right)$ will not. Since $\operatorname{Pr}\left(x_{i}<\underline{x}_{1}^{j *} \mid \theta\right)=$ $\Phi\left(\sqrt{\beta^{j}}\left(\underline{x}_{1}^{j *}-\theta\right)\right)$ and $\operatorname{Pr}\left(x_{i}<\bar{x}_{1}^{j *} \mid \theta\right)=\Phi\left(\sqrt{\beta^{j}}\left(\bar{x}_{1}^{j *}-\theta\right)\right)$ for fixed $\underline{x}_{1}^{j *}$ and $\bar{x}_{1}^{j *}$, speculators must believe, even under the most pessimistic belief about the success of attack against $j$, that $j$ abandons the peg for $\theta^{j} \leq \underline{\theta}_{2}^{j *}\left(\theta^{-j}\right)$, and even under the most optimistic belief about the success of attack against $j$, that $j$ sustains the peg for $\theta^{j}>\bar{\theta}_{2}^{j *}\left(\theta^{-j}\right)$, where

$$
\begin{aligned}
& \underline{\theta}_{2}^{j *}\left(\theta^{-j}\right)=\int_{\epsilon_{i}^{-j} \in \mathbb{R}^{N-1}} \tilde{\phi}^{-j}\left(\epsilon_{i}^{-j}\right) \Phi\left(\sqrt{\beta^{j}}\left(\underline{x}_{1}^{j *}\left(\theta^{-j}+\epsilon_{i}^{-j}\right)-\underline{\theta}_{2}^{j *}\left(\theta^{-j}\right)\right)\right) d \epsilon_{i}^{-j}, \\
& \bar{\theta}_{2}^{j *}\left(\theta^{-j}\right)=\int_{\epsilon_{i}^{-j} \in \mathbb{R}^{N-1}} \tilde{\phi}^{-j}\left(\epsilon_{i}^{-j}\right) \Phi\left(\sqrt{\beta^{j}}\left(\bar{x}_{1}^{j *}\left(\theta^{-j}+\epsilon_{i}^{-j}\right)-\bar{\theta}_{2}^{j *}\left(\theta^{-j}\right)\right)\right) d \epsilon_{i}^{-j} .
\end{aligned}
$$

Clearly, $1=\bar{\theta}_{1}^{j *}\left(\theta^{-j}\right) \geq \bar{\theta}_{2}^{j *}\left(\theta^{-j}\right) \geq \underline{\theta}_{2}^{j *}\left(\theta^{-j}\right) \geq \underline{\theta}_{1}^{j *}\left(\theta^{-j}\right)=0$ for all $\theta^{-j}$.

Repeating this procedure, one recursively obtains the sequences of functions $\left\{\underline{\Gamma}_{n}^{j *}, \bar{\Gamma}_{n}^{j *}, \underline{x}_{n}^{j *}, \bar{x}_{n}^{j *}, \underline{\theta}_{n+1}^{j *}, \bar{\theta}_{n+1}^{j *}\right\}_{n=1}^{\infty}$ as

$$
\begin{aligned}
\underline{\Gamma}_{n}^{j *}\left(x_{i}\right) & =\int_{\epsilon_{i}^{-j} \in \mathbb{R}^{N-1}} \tilde{\phi}^{-j}\left(\epsilon_{i}^{-j}\right) \Phi\left(\sqrt{\beta^{j}}\left(\underline{\theta}_{n}^{j *}\left(x_{i}^{-j}-\epsilon_{i}^{-j}\right)-x_{i}^{j}\right)\right) d \epsilon_{i}^{-j}-c^{j}, \\
\bar{\Gamma}_{n}^{j *}\left(x_{i}\right) & =\int_{\epsilon_{i}^{-j} \in \mathbb{R}^{N-1}} \tilde{\phi}^{-j}\left(\epsilon_{i}^{-j}\right) \Phi\left(\sqrt{\beta^{j}}\left(\bar{\theta}_{n}^{j *}\left(x_{i}^{-j}-\epsilon_{i}^{-j}\right)-x_{i}^{j}\right)\right) d \epsilon_{i}^{-j}-c^{j}, \\
\underline{x}_{n}^{j *}\left(x_{i}^{-j}\right) & =\inf \left\{x_{i}^{j} \mid \underline{\Gamma}_{n}^{j *}\left(x_{i}\right) \leq \max \left\{0,\left(\bar{\Gamma}_{n}^{k *}\left(x_{i}\right)\right)_{k \neq j}\right\}\right\} \text { if inf exists, } \\
& =-\infty \text { otherwise, } \\
\bar{x}_{n}^{j *}\left(x_{i}^{-j}\right) & =\sup \left\{x_{i}^{j} \mid \bar{\Gamma}_{n}^{j *}\left(x_{i}\right) \geq \max \left\{0,\left(\underline{\Gamma}_{n}^{k *}\left(x_{i}\right)\right)_{k \neq j}\right\}\right\} \text { if sup exists, } \\
& =-\infty \text { otherwise, } \\
\underline{\theta}_{n+1}^{j *}\left(\theta^{-j}\right) & =\int_{\epsilon_{i}^{-j} \in \mathbb{R}^{N-1}} \tilde{\phi}^{-j}\left(\epsilon_{i}^{-j}\right) \Phi\left(\sqrt{\beta^{j}}\left(\underline{x}_{n}^{j *}\left(\theta^{-j}+\epsilon_{i}^{-j}\right)-\underline{\theta}_{n+1}^{j *}\left(\theta^{-j}\right)\right)\right) d \epsilon_{i}^{-j}, \\
\bar{\theta}_{n+1}^{j *}\left(\theta^{-j}\right) & =\int_{\epsilon_{i}^{-j} \in \mathbb{R}^{N-1}} \tilde{\phi}^{-j}\left(\epsilon_{i}^{-j}\right) \Phi\left(\sqrt{\beta^{j}}\left(\bar{x}_{n}^{j *}\left(\theta^{-j}+\epsilon_{i}^{-j}\right)-\bar{\theta}_{n+1}^{j *}\left(\theta^{-j}\right)\right)\right) d \epsilon_{i}^{-j} .
\end{aligned}
$$

${ }^{11}$ The supremum may not exist when $c^{j}>c^{k}$, since for sufficiently small $x_{i}^{k}, \underline{\Gamma}_{1}^{k *}\left(x_{i}\right)=\Phi\left(-x_{i}^{k}\right)-c^{k}>$ $1-c^{j}=\lim _{x_{i}^{j} \rightarrow-\infty} \bar{\Gamma}_{1}^{j *}\left(x_{i}\right)$, hence the set $\left\{x_{i}^{j} \mid \bar{\Gamma}_{1}^{j *}\left(x_{i}\right) \geq \max \left\{0,\left(\underline{\Gamma}_{1}^{k *}\left(x_{i}\right)_{k \neq j}\right)\right\}\right\}$ is empty. The opposite case where $\bar{\Gamma}_{1}^{j *}\left(x_{i}\right) \geq \max \left\{0,\left(\underline{\Gamma}_{1}^{k *}\left(x_{i}\right)_{k \neq j}\right)\right\}$ for arbitrarily large $x_{i}^{j}$ does not arise, since $\bar{\Gamma}_{1}^{j *}\left(x_{i}\right)$ decreases continuously in $x_{i}^{j}$ with $\lim _{x_{i}^{j} \rightarrow \infty} \bar{\Gamma}_{1}^{j *}\left(x_{i}\right)=-c^{j}<0$. The same applies to subsequent rounds. 
For any $X \subseteq \mathbb{R}^{l}$ and $g, g^{\prime}: X \rightarrow \mathbb{R}$, write $g \geq g^{\prime}$ to imply $g(x) \geq g^{\prime}(x)$ for any $x \in X$. Then, given $\bar{\theta}_{1}^{j *} \geq \bar{\theta}_{2}^{j *} \geq \underline{\theta}_{2}^{j *} \geq \underline{\theta}_{1}^{j *},(17)$ and (18) imply $\bar{\Gamma}_{1}^{j *} \geq \bar{\Gamma}_{2}^{j *} \geq \underline{\Gamma}_{2}^{j *} \geq \underline{\Gamma}_{1}^{j *}$, which in turn implies $\bar{x}_{1}^{j *} \geq \bar{x}_{2}^{j *} \geq \underline{x}_{2}^{j *} \geq \underline{x}_{1}^{j *}$ from (19) and (20). But then, (21) and (22) imply $\bar{\theta}_{2}^{j *} \geq \bar{\theta}_{3}^{j *} \geq \underline{\theta}_{3}^{j *} \geq \underline{\theta}_{2}^{j *}$. Proceeding in the same fashion, it follows that for $j=\{1,2, \ldots, N\},\left\{\underline{\theta}_{n}^{j *}, \underline{\Gamma}_{n}^{j *}, \underline{x}_{n}^{j *}\right\}_{n=1}^{\infty}$ are nondecreasing sequences and $\left\{\bar{\theta}_{n}^{j *}, \bar{\Gamma}_{n}^{j *}, \bar{x}_{n}^{j *}\right\}_{n=1}^{\infty}$ are nonincreasing sequences, where $1 \geq \bar{\theta}_{n}^{j *} \geq \underline{\theta}_{n}^{j *} \geq 0, \bar{\Gamma}_{n}^{j *} \geq \underline{\Gamma}_{n}^{j *}$, and $\bar{x}_{n}^{j *} \geq \underline{x}_{n}^{j *}$ for all $n$.

There are several points that should be noted about the procedure described above. First, $\underline{\Gamma}_{n}^{j *}\left(x_{i}\right)$ and $\bar{\Gamma}_{n}^{j *}\left(x_{i}\right)$ are always decreasing in $x_{i}^{j}$, and so are $\underline{\Gamma}_{n}^{j *}\left(x_{i}\right)-\bar{\Gamma}_{n}^{k *}\left(x_{i}\right)$ and $\bar{\Gamma}_{n}^{j *}\left(x_{i}\right)-\underline{\Gamma}_{n}^{k *}\left(x_{i}\right)$ when $\underline{\theta}_{n}^{j *}$ and $\bar{\theta}_{n}^{j *}$ are nondecreasing. In such case, there is no $x_{i}^{j} \geq$ $\underline{x}_{n}^{j *}\left(x_{i}^{-j}\right)$ such that $\underline{\Gamma}_{n}^{j *}\left(x_{i}\right)>\max \left\{0,\left(\bar{\Gamma}_{n}^{k *}\left(x_{i}\right)\right)_{k \neq j}\right\}$ and no $x_{i}^{j} \leq \bar{x}_{n}^{j *}\left(x_{i}^{-j}\right)$ such that $\bar{\Gamma}_{n}^{j *}\left(x_{i}\right)<\max \left\{0,\left(\underline{\Gamma}_{n}^{k *}\left(x_{i}\right)\right)_{k \neq j}\right\}$, so in each round, all strictly dominated strategies are eliminated. When $\underline{\theta}_{n}^{j *}$ and $\bar{\theta}_{n}^{j *}$ are not necessarily nondecreasing, however, some strictly dominated strategies may be left uneliminated in each round. This is not an issue of concern, since the order of deletion of strictly (as opposed to weakly) dominated strategies is irrelevant. Second, while the procedure above is a systematic method for deleting strictly dominated strategies, it is not the only possible procedure, since the order of deletion is irrelevant. What is critical is to eliminate only strictly dominated strategies, and the definitions of $\underline{x}_{n}^{j *}$ and $\bar{x}_{n}^{j *}$ in (19) and (20) guarantee that this is the case, even when $\underline{\theta}_{n}^{j *}$ and $\bar{\theta}_{n}^{j *}$ are not continuous and nondecreasing.

For each $\theta^{-j} \in \mathbb{R}^{N-1},\left\{\underline{\theta}_{n}^{j *}\left(\theta^{-j}\right)\right\}_{n=1}^{\infty}$ is a nondecreasing sequence in $\mathbb{R}$, and $\left\{\bar{\theta}_{n}^{j *}\left(\theta^{-j}\right)\right\}_{n=1}^{\infty}$ is a nonincreasing sequence in $\mathbb{R}$, where each sequence is, respectively, bounded above and below by the other. Thus, the sequences of functions $\left\{\underline{\theta}_{n}^{j *}\right\}_{n=1}^{\infty}$ and $\left\{\bar{\theta}_{n}^{j *}\right\}_{n=1}^{\infty}$ each converge pointwise. However, this argument is insufficient to adequately explore the issues of main interest, such as the existence and uniqueness of equilibrium threshold functions. These issues are addressed by resorting to the contraction mapping theorem.

\subsection{Contraction Mapping}

The equations (17)-(22) map $2 N$ functions $\left\{\underline{\theta}_{n}^{j *}, \bar{\theta}_{n}^{j *}\right\}_{j=1}^{N}$ to $\left\{\underline{\theta}_{n+1}^{j *}, \bar{\theta}_{n+1}^{j *}\right\}_{j=1}^{N}$. One must define an operator representing this mapping on an adequate space, such that the contraction mapping theorem can be applied.

For any $X \subseteq \mathbb{R}^{l}$, let $B\left(X, \mathbb{R}^{N}\right)$ denote the space of bounded, continuous, vectorvalued functions $g: X \rightarrow \mathbb{R}^{N}, g=\left(g^{1}, g^{2}, \ldots, g^{N}\right)$, equipped with the max-sup norm $\|g\| \equiv \max _{j=1}^{N}\left(\sup _{z \in X}\left|g^{j}(z)\right|\right)$. Further, let $\tilde{B}\left(X, \mathbb{R}^{N}\right)$ denote the space of bounded, continuous, nondecreasing vector-valued functions $g: X \rightarrow \mathbb{R}^{N}, g=\left(g^{1}, g^{2}, \ldots, g^{N}\right)$, with the same norm. Then, $\tilde{B}\left(X, \mathbb{R}^{N}\right)$ is a closed subset of $B\left(X, \mathbb{R}^{N}\right)$.

Now, define vector-valued functions $\underline{\theta}_{n}^{*} \equiv\left(\underline{\theta}_{n}^{1 *}, \underline{\theta}_{n}^{2 *}, \ldots, \underline{\theta}_{n}^{N *}\right)$ and $\bar{\theta}_{n}^{*} \equiv\left(\bar{\theta}_{n}^{1 *}, \bar{\theta}_{n}^{2 *}, \ldots, \bar{\theta}_{n}^{N *}\right)$, 
and an operator $T$ on $\tilde{B}\left(\mathbb{R}^{N}, \mathbb{R}^{2 N}\right)$ by $g_{n+1}^{*}=T g_{n}^{*}$, where $g_{n}^{*}=\left(\underline{\theta}_{n}^{*}, \bar{\theta}_{n}^{*}\right) .{ }^{12}$ In other words, ${ }^{13}$

$$
g_{n}^{*}(\theta)=\left(\underline{\theta}_{n}^{1 *}\left(\theta^{-1}\right), \ldots, \underline{\theta}_{n}^{N *}\left(\theta^{-N}\right), \bar{\theta}_{n}^{1 *}\left(\theta^{-1}\right), \ldots, \bar{\theta}_{n}^{N *}\left(\theta^{-N}\right)\right) .
$$

The max-sup norm applied to such $g_{n}^{*}$ is

$$
\left\|g_{n}^{*}\right\| \equiv \max _{j=1}^{N}\left[\max \left(\sup _{\theta^{-j} \in \mathbb{R}^{N-1}}\left|\underline{\theta}_{n}^{j *}\left(\theta^{-j}\right)\right|, \sup _{\theta^{-j} \in \mathbb{R}^{N-1}}\left|\bar{\theta}_{n}^{j *}\left(\theta^{-j}\right)\right|\right)\right] .
$$

If $\tilde{B}\left(\mathbb{R}^{N}, \mathbb{R}^{2 N}\right)$ is a complete metric space, $T: \tilde{B}\left(\mathbb{R}^{N}, \mathbb{R}^{2 N}\right) \rightarrow \tilde{B}\left(\mathbb{R}^{N}, \mathbb{R}^{2 N}\right)$, and $T$ is a contraction, then the contraction mapping theorem implies that $T$ has a unique fixed point in $\tilde{B}\left(\mathbb{R}^{N}, \mathbb{R}^{2 N}\right)$. Let $\left(\underline{\theta}^{*}, \bar{\theta}^{*}\right)$ denote this fixed point, and $\underline{x}^{*}=\left(\underline{x}^{1 *}, \underline{x}^{2 *}, \ldots, \underline{x}^{N *}\right), \bar{x}^{*}=$ $\left(\bar{x}^{1 *}, \bar{x}^{2 *}, \ldots, \bar{x}^{N *}\right)$ be the associated threshold signal functions. Since the theorem also implies that $g_{n}^{*}$ converges to this fixed point from any $g_{0}^{*} \in \tilde{B}\left(\mathbb{R}^{N}, \mathbb{R}^{2 N}\right)$, this fixed point corresponds to the limiting functions from the iterative deletion of dominated strategies.

Once this step is accomplished, it remains to show $\underline{\theta}^{*}=\bar{\theta}^{*}$; this implies $\underline{x}^{*}=\bar{x}^{*}$, such that speculator $i$ attacks currency $j$ if $x_{i}^{j}<x^{j *}\left(x_{i}^{-j}\right)$, and never does so if $x_{i}^{j}>x^{j *}\left(x_{i}^{-j}\right)$. That $\underline{\theta}^{*}=\bar{\theta}^{*}$ can be established by showing that $T$ maps $g_{n}^{*}=\left(\underline{\theta}_{n}^{*}, \bar{\theta}_{n}^{*}\right)$ with $\underline{\theta}_{n}^{*}=\bar{\theta}_{n}^{*}$ to $g_{n+1}^{*}=\left(\underline{\theta}_{n+1}^{*}, \bar{\theta}_{n+1}^{*}\right)$ with $\underline{\theta}_{n+1}^{*}=\bar{\theta}_{n+1}^{*}$, and invoking the corollary to the contraction mapping theorem.

Thus, the key is to show that $T$ satisfies the requirement of the contraction mapping theorem. It is straightforward to show that $\tilde{B}\left(\mathbb{R}^{N}, \mathbb{R}^{2 N}\right)$ is a complete metric space. For $N=2$, one can further show that $T: \tilde{B}\left(\mathbb{R}^{N}, \mathbb{R}^{2 N}\right) \rightarrow \tilde{B}\left(\mathbb{R}^{N}, \mathbb{R}^{2 N}\right)$ and that $T$ is a contraction mapping. For $N>2$, this can be shown only for the symmetric target case, and requires a slightly modified approach. The next two subsections examine these cases in turn, and discuss the resulting equilibrium.

\subsection{Equilibrium with $N=2$ Targets}

For $N=2$, one can show that iterative deletion of dominated strategies yields a unique combination of equilibrium threshold functions, as summarized below. ${ }^{14}$

Proposition 1. For $N=2$, the equilibrium is characterized by a unique combination of threshold signal functions $x^{j *}$ and threshold fundamentals functions $\theta^{j *}, j=\{1,2\}$, such that

\footnotetext{
${ }^{12}$ The reason for defining an operator on threshold fundamentals functions, not threshold signal functions, is that, unlike the former, which is guaranteed to have values in $[0,1]$, the latter is not bounded.

${ }^{13}$ Alternatively, I could define $\underline{\theta}_{n}^{j *}$ and $\bar{\theta}_{n}^{*}$ as functions of $\theta \in \mathbb{R}^{N}$, where $\underline{\theta}_{n}^{j *}$ and $\bar{\theta}_{n}^{*}$ are invariant to $\theta^{j}$. Such formulation simplifies exposition but obscures the fact that $\underline{\theta}_{n}^{j *}$ and $\bar{\theta}_{n}^{*}$ depend only on $\theta^{-j}$, which is why the current formulation is chosen. In any case, these choices are not essential to the results.

${ }^{14}$ The proofs to all propositions are in the appendix.
} 
1. A speculator attacks currency $j$ if $x_{i}^{j}<x^{j *}\left(x_{i}^{-j}\right)$ and only if $x_{i}^{j} \leq x^{j *}\left(x_{i}^{-j}\right)$, where $x^{j *}$ is continuous and increasing.

2. Country $j$ abandons the peg if and only if $\theta^{j} \leq \theta^{j *}\left(\theta^{-j}\right)$, where $\theta^{j *} \in(0,1)$ and $\theta^{j *}$ is continuous and increasing.

The proof proceeds exactly as described in Sections 3.2 and 3.3; that is, by resorting to the contraction mapping theorem to establish the existence and uniqueness of the fixed point of $T$, and by resorting to the corollary of this theorem to show that the lower and upper threshold functions coincide at this fixed point, and to obtain additional properties of the equilibrium functions.

Proposition 1 completely specifies the speculators' attacking strategy, except when $x_{i}^{j}=x^{j *}\left(x_{i}^{-j}\right)$. This corresponds to the case in which the speculator is indifferent between attacking $j$, and either attacking some other currency or not attacking. Therefore, once the tie-breaking rule is specified, iterative deletion of dominated strategies leads to a unique equilibrium. Since marginal speculators with signals $x_{i}^{j}=x^{j *}\left(x_{i}^{-j}\right)$ have measure zero, such tie-breaking rule has no impact on the outcomes of attack; the combination of fundamentals $\left(\theta^{1}, \theta^{2}\right)$ uniquely pins down whether countries 1 and 2 sustain or abandon the peg. For the same reason, focusing on pure strategy and ruling out partial attacks is also immaterial.

Note that in the equilibrium above, the threshold fundamentals and the threshold signals of one country are affected by the fundamentals and signals of the other country, even though neither the fundamentals nor the signals of the two countries are correlated. More precisely, a speculator is more willing to attack currency $j$ when that speculator's signal for the other country is strong, and country $j$ is more likely to abandon the peg when the other country has strong fundamentals.

\subsection{Equilibrium with Symmetric $N>2$ Targets}

For $N>2$, the argument above does not apply, at least immediately, for the following reason. Recall the operator $T$ defined by (17)-(22). When $\underline{\theta}_{n}^{j *}$ and $\bar{\theta}_{n}^{j *}$ are bounded, continuous and nondecreasing, it is possible to show that the Blackwell's sufficient conditions for contraction, namely monotonicity and discounting, are satisfied. What cannot be shown is that $\underline{\theta}_{n+1}^{j *}$ and $\bar{\theta}_{n+1}^{j *}$ are again nondecreasing. To see this, consider the case of $N=3$, and take $\left(x_{i}^{2}, x_{i}^{3}\right)$ such that $\underline{x}_{n}^{1 *}\left(x_{i}^{2}, x_{i}^{3}\right)$ is given by the condition

$$
\underline{\Gamma}_{n}^{1 *}\left(\underline{x}_{n}^{1 *}\left(x_{i}^{2}, x_{i}^{3}\right), x_{i}^{2}, x_{i}^{3}\right)=\bar{\Gamma}_{n}^{2 *}\left(\underline{x}_{n}^{1 *}\left(x_{i}^{2}, x_{i}^{3}\right), x_{i}^{2}, x_{i}^{3}\right)>\max \left\{0, \bar{\Gamma}_{n}^{3 *}\left(\underline{x}_{n}^{1 *}\left(x_{i}^{2}, x_{i}^{3}\right), x_{i}^{2}, x_{i}^{3}\right)\right\} .
$$


Now, take $\Delta>0$ sufficiently small, and consider $\underline{x}_{n}^{1 *}\left(x_{i}^{2}, x_{i}^{3}+\Delta\right)$. Since $\underline{\theta}_{n}^{j *}$ and $\bar{\theta}_{n}^{j *}$ are continuous by assumption, so are $\underline{\Gamma}_{n}^{j *}$ and $\bar{\Gamma}_{n}^{j *}$. Then, $\underline{x}_{n}^{1 *}\left(x_{i}^{2}, x_{i}^{3}+\Delta\right)$ is still determined by the indifference condition between attacking 1 and 2 ,

$$
\underline{\Gamma}_{n}^{1 *}\left(\underline{x}_{n}^{1 *}\left(x_{i}^{2}, x_{i}^{3}+\Delta\right), x_{i}^{2}, x_{i}^{3}+\Delta\right)=\bar{\Gamma}_{n}^{2 *}\left(\underline{x}_{n}^{1 *}\left(x_{i}^{2}, x_{i}^{3}+\Delta\right), x_{i}^{2}, x_{i}^{3}+\Delta\right) .
$$

As $x_{i}^{3}$ rises to $x_{i}^{3}+\Delta, \underline{\Gamma}_{n}^{1 *}$ and $\bar{\Gamma}_{n}^{2 *}$ weakly increase since $\underline{\theta}_{n}^{j *}$ and $\bar{\theta}_{n}^{j *}$ are nondecreasing by assumption. To restore equality, $x_{i}^{1}$ may need to rise or fall, so it is possible that $\underline{x}_{n}^{1 *}\left(x_{i}^{2}, x_{i}^{3}+\Delta\right)<\underline{x}_{n}^{1 *}\left(x_{i}^{2}, x_{i}^{3}\right)$. In other words, while $\underline{x}_{n}^{1 *}\left(x_{i}^{2}, x_{i}^{3}\right)$ defined by $(25)$ is increasing in $x_{i}^{2}$, it need not be nondecreasing in $x_{i}^{3}$. But then, $(21)$ implies that $\underline{\theta}_{n+1}^{1 *}\left(\theta^{2}, \theta^{3}\right)$ may not be nondecreasing in $\theta^{3}$.

More generally, the argument above implies that for $N>2, \underline{x}_{n}^{j *}$ and $\bar{x}_{n}^{j *}$ need not be nondecreasing, which in turn implies that $\underline{\theta}_{n+1}^{j *}$ and $\bar{\theta}_{n+1}^{j *}$ may not be nondecreasing even if $\underline{\theta}_{n}^{j *}$ and $\bar{\theta}_{n}^{j *}$ are so. ${ }^{15}$ Thus, $T$ cannot be shown to be a self-map on $\tilde{B}\left(\mathbb{R}^{N}, \mathbb{R}^{2 N}\right)$. Defining $T$ instead on $B\left(\mathbb{R}^{N}, \mathbb{R}^{2 N}\right)$ does not resolve the issue, because then $T$ turns out to lose the discounting property.

This issue can be overcome for the symmetric case in which $\beta^{j}$ and $c^{j}$ are the same across $j$. The trick is to slightly modify the procedure of iterative deletion as follows. In the $n$-th round of iteration, compute $\underline{\Gamma}_{n}^{j *}$ and $\bar{\Gamma}_{n}^{j *}$ from $\underline{\theta}_{n}^{j *}$ and $\bar{\theta}_{n}^{j *}$ by (17) and (18), and then $\underline{x}_{n}^{j *}$ and $\bar{x}_{n}^{j *}$ by (19) and (20). Now, define the sets of functions in $\tilde{B}\left(\mathbb{R}^{N-1}, \mathbb{R}\right)$ that is dominated by $\underline{x}_{n}^{j *}$, and that dominates $\bar{x}_{n}^{j *}$, by

$$
\begin{aligned}
& \underline{X}_{n}^{j *} \equiv\left\{f \in \tilde{B}\left(\mathbb{R}^{N-1}, \mathbb{R}\right) \mid f \leq \underline{x}_{n}^{j *}\right\}, \\
& \bar{X}_{n}^{j *} \equiv\left\{g \in \tilde{B}\left(\mathbb{R}^{N-1}, \mathbb{R}\right) \mid g \geq \bar{x}_{n}^{j *}\right\} .
\end{aligned}
$$

Then, define functions $\underline{x}_{n, N D}^{j *}$ and $\bar{x}_{n, N D}^{j *}$ as

$$
\begin{aligned}
& \underline{x}_{n, N D}^{j *}=\left\{f \in \underline{X}_{n}^{j *} \mid f \geq f^{\prime}, \forall f^{\prime} \in \underline{X}_{n}^{j *}\right\}, \\
& \bar{x}_{n, N D}^{j *}=\left\{g \in \bar{X}_{n}^{j *} \mid g \leq g^{\prime}, \forall g^{\prime} \in \bar{X}_{n}^{j *}\right\} .
\end{aligned}
$$

Finally, define $\underline{\theta}_{n+1}^{j *}$ and $\bar{\theta}_{n+1}^{j *}$ by

$$
\begin{aligned}
& \underline{\theta}_{n+1}^{j *}\left(\theta^{-j}\right)=\int_{\epsilon_{i}^{-j} \in \mathbb{R}^{N-1}} \tilde{\phi}^{-j}\left(\epsilon_{i}^{-j}\right) \Phi\left(\sqrt{\beta^{j}}\left(\underline{x}_{n, N D}^{j *}\left(\theta^{-j}+\epsilon_{i}^{-j}\right)-\underline{\theta}_{n+1}^{j *}\left(\theta^{-j}\right)\right)\right) d \epsilon_{i}^{-j}, \\
& \bar{\theta}_{n+1}^{j *}\left(\theta^{-j}\right)=\int_{\epsilon_{i}^{-j} \in \mathbb{R}^{N-1}} \tilde{\phi}^{-j}\left(\epsilon_{i}^{-j}\right) \Phi\left(\sqrt{\beta^{j}}\left(\bar{x}_{n, N D}^{j *}\left(\theta^{-j}+\epsilon_{i}^{-j}\right)-\bar{\theta}_{n+1}^{j *}\left(\theta^{-j}\right)\right)\right) d \epsilon_{i}^{-j} .
\end{aligned}
$$

\footnotetext{
${ }^{15}$ Note that this problem does not arise for $N=2$. If $\underline{\theta}_{n}^{j *}$ and $\bar{\theta}_{n}^{j *}$ are nondecreasing, $\underline{\Gamma}_{n}^{1 *}\left(x_{i}^{1}, x_{i}^{2}\right)$ is decreasing in $x_{i}^{1}$ and nondecreasing in $x_{i}^{2}$, whereas $\bar{\Gamma}_{n}^{2 *}\left(x_{i}^{1}, x_{i}^{2}\right)$ is decreasing in $x_{i}^{2}$ and nondecreasing in $x_{i}^{1}$. Then, $\underline{x}_{n}^{1 *}\left(x_{i}^{2}\right)$ is nondecreasing in $x_{i}^{2}$, hence $\underline{\theta}_{n+1}^{1 *}\left(\theta^{2}\right)$ is nondecreasing in $\theta^{2}$.
} 
Let $T^{\prime}$ denote this modified operator defined by (17)-(20) and (27)-(32), which maps from $g_{n}^{*}=\left(\underline{\theta}_{n}^{*}, \bar{\theta}_{n}^{*}\right)$ to $g_{n+1}^{*}=\left(\underline{\theta}_{n+1}^{*}, \bar{\theta}_{n+1}^{*}\right)$.

This modification forces $\underline{\theta}_{n+1}^{j *}$ and $\bar{\theta}_{n+1}^{j *}$ to be nondecreasing, by eliminating the strategies 'do not attack currency $j$ ' only for $x_{i}^{j}<\underline{x}_{n, N D}^{j *}\left(x_{i}^{-j}\right) \leq \underline{x}_{n}^{j *}\left(x_{i}^{-j}\right)$, and 'attack currency $j$ ' only for $x_{i}^{j}>\bar{x}_{n, N D}^{j *}\left(x_{i}^{-j}\right) \geq \bar{x}_{n}^{j *}\left(x_{i}^{-j}\right)$, where $\underline{x}_{n, N D}^{j *}$ and $\bar{x}_{n, N D}^{j *}$ are nondecreasing. Since one must avoid eliminating strategies that are not strictly dominated, choosing $\underline{x}_{n, N D}^{j *} \in \underline{X}_{n}^{j *}$ and $\bar{x}_{n, N D}^{j *} \in \bar{X}_{n}^{j *}$ is critical. On the other hand, choosing $\underline{x}_{n, N D}^{j *}$ and $\bar{x}_{n, N D}^{j *}$ to be the largest and smallest function, respectively, in $\underline{X}_{n}^{j *}$ and $\bar{X}_{n}^{j *}$ is inessential, because the order of deletion of strictly dominated strategies is irrelevant. This can also be seen from the fact that when the contraction mapping theorem applies, the fixed point is achieved from any initial value. Then, one can show that the operator $T^{\prime}$ is a contraction on $\tilde{B}\left(\mathbb{R}^{N}, \mathbb{R}^{2 N}\right)$, and hence has a unique fixed point, as stated in the following proposition.

Proposition 2. $T^{\prime}$ has a unique fixed point, $\left(\underline{\theta}^{*}, \bar{\theta}^{*}\right)$.

The final step is to show $\underline{\theta}^{*}=\bar{\theta}^{*}$, which is achieved by taking advantage of the symmetry across targets, namely that $\beta^{j}$ and $c^{j}$ are common to all $j$. The following definition is introduced to facilitate the discussion.

Definition. $h^{j}: \mathbb{R}^{N} \rightarrow \mathbb{R}, j=\{1,2, \ldots, N\}$, are said to be symmetric across $j$ if for any $s=\left(s_{1}, s_{2}, \ldots, s_{N}\right)$ and $j, k \in\{1,2, \ldots, N\},(1) h^{j}(s)=h^{k}\left(s^{\prime}\right)$ where $s^{\prime}$ is created from $s$ by exchanging its $j$-th and $k$-th element, and (2) $h^{j}\left(s^{j}, s^{-j}\right)=h^{j}\left(s^{j}, \tilde{s}^{-j}\right)$ for any permutation $\tilde{s}^{-j}=\left(\tilde{s}_{1}, \ldots, \tilde{s}_{j-1}, \tilde{s}_{j+1}, \ldots, \tilde{s}_{N}\right)$ of $\left\{s_{1}, \ldots, s_{j-1}, s_{j+1}, \ldots, s_{N}\right\}$.

For $h^{j}: \mathbb{R}^{N-1} \rightarrow \mathbb{R}, j=\{1,2, \ldots, N\}$, which are functions only of $s^{-j}$, the definition above can be applied by considering $h^{j}$ as functions of $s \in \mathbb{R}^{N}$, whose values do not vary with $s^{j}{ }^{16}$ In the present context, that $h^{j}$ is symmetric across $j$ simply implies that the roles played by $N$ countries are identical. Then, one can show that $T^{\prime}$ maps $g_{n}^{*}=\left(\underline{\theta}_{n}^{*}, \bar{\theta}_{n}^{*}\right) \in \tilde{B}\left(\mathbb{R}^{N}, \mathbb{R}^{2 N}\right)$, where $\underline{\theta}_{n}^{*}=\bar{\theta}_{n}^{*} \equiv \theta_{n}^{*}=\left(\theta_{n}^{1 *}, \theta_{n}^{2 *}, \ldots, \theta_{n}^{N *}\right)$ with $\theta_{n}^{j *}$ symmetric across $j$, to $g_{n+1}^{*}=\left(\underline{\theta}_{n+1}^{*}, \bar{\theta}_{n+1}^{*}\right)$ with the same properties. Further, for such $g_{n}^{*}$, one can also show that $\underline{x}_{n}^{j *}=\bar{x}_{n}^{j *}$ and that these are nondecreasing, which implies $\underline{x}_{n}^{j *}=\bar{x}_{n}^{j *}=$ $\underline{x}_{n, N D}^{j *}=\bar{x}_{n, N D}^{j *}$. It then follows that with symmetric targets, the unique fixed point of $T^{\prime}$ is such that $\underline{\theta}^{*}=\bar{\theta}^{*} \equiv \theta^{*}$, which yields $\underline{x}^{*}=\bar{x}^{*} \equiv x^{*}$. But since the set of eliminated strategies each round by the procedure corresponding to operator $T^{\prime}$ is a subset of that associated with operator $T$, the unique fixed point of $T^{\prime}$ must coincide with that of $T$, or equivalently, with the limiting function from the iterative deletion of dominated strategies. This argument establishes the following results.

\footnotetext{
${ }^{16}$ For example, let $N=3$ and consider $\underline{\theta}_{n}^{1 *}\left(\theta^{2}, \theta^{3}\right), \underline{\theta}_{n}^{2 *}\left(\theta^{1}, \theta^{3}\right), \underline{\theta}_{n}^{3 *}\left(\theta^{1}, \theta^{2}\right)$. If $\underline{\theta}_{n}^{j *}$ is symmetric across $j$, then for any $a, b \in \mathbb{R}, \underline{\theta}_{n}^{j *}(a, b)=\underline{\theta}_{n}^{j *}(b, a)$ for all $j$, and the value is independent of $j$.
} 
Proposition 3. For $N>2$, suppose $\beta^{j}=\beta>0$ and $c^{j}=c \in(0,1)$ for all $j \in$ $\{1,2, \ldots, N\}$. Then the equilibrium is characterized by a unique combination of threshold signal functions $x^{j *}$ and threshold fundamentals functions $\theta^{j *}$ that are symmetric across $j$, such that

1. A speculator attacks currency $j$ if $x_{i}^{j}<x^{j *}\left(x_{i}^{-j}\right)$ and only if $x_{i}^{j} \leq x^{j *}\left(x_{i}^{-j}\right)$, where $x^{j *}$ is continuous and nondecreasing, and $x^{j *}\left(x_{i}^{-j}\right) \leq \min _{k \neq j} x_{i}^{k}$.

2. Country $j$ abandons the peg if and only if $\theta^{j} \leq \theta^{j *}\left(\theta^{-j}\right)$, where $\theta^{j *} \in(0,1)$ and $\theta^{j *}$ is continuous and increasing.

Proposition 3 implies, as in the case of $N=2$, that the equilibrium is unique up to ties and that the outcomes of attack for the $N$ countries are uniquely pinned down given the fundamentals of all countries. Further, the equilibrium strategy has an intuitive property; $x^{j *}\left(x_{i}^{-j}\right) \leq \min _{k \neq j} x_{i}^{k}$ implies that if a speculator attacks, the speculator attacks the currency with the lowest signal.

This approach is, however, silent on the existence and uniqueness of equilibrium threshold functions in environments with $N>2$ nonsymmetric targets. The argument for establishing existence and uniqueness of the fixed point $\left(\underline{\theta}^{*}, \bar{\theta}^{*}\right)$ of $T^{\prime}$ does not hinge on targets being symmetric. However, with nonsymmetric targets, $\underline{\theta}_{n}^{*}=\bar{\theta}_{n}^{*}$ does not imply $\underline{\theta}_{n+1}^{*}=\bar{\theta}_{n+1}^{*}$. This is because, as observed from (29) and (30), $\underline{x}_{n, N D}^{j *} \neq \bar{x}_{n, N D}^{j *}$ even if $\underline{x}_{n}^{j *}=\bar{x}_{n}^{j *}$, unless $\underline{x}_{n}^{j *}\left(=\bar{x}_{n}^{j *}\right)$ is nondecreasing, which cannot be guaranteed to be the case. Accordingly, the final step of establishing $\underline{\theta}^{*}=\bar{\theta}^{*}$ cannot be accomplished.

\section{Numerical Examples}

It is shown above that for $N=2$ targets, as well as for general $N$ symmetric targets, the equilibrium is characterized by threshold functions that are uniquely determined. Unlike the threshold values of fundamentals and signal in the single-target case discussed in Section 2.2.2, however, these functions cannot be obtained in closed form. This section explores several numerical examples, in order to obtain additional insights on these equilibrium functions. ${ }^{17}$

\subsection{Symmetric and Nonsymmetric $N=2$ Targets}

This subsection discusses the case of $N=2$, which is the main focus of the analysis.

\footnotetext{
${ }^{17}$ Detailed computational procedures are described in the appendix.
} 


\subsubsection{Symmetric Targets}

I first consider the case with common $c^{j}$ and $\beta^{j}$. Note that results are symmetric across two countries in this case, hence the explanations below apply when the roles of country 1 and 2 are reversed. Figures $1-4$ illustrate the equilibrium threshold functions for $c^{1}=c^{2}=c=0.1$ and $\beta^{1}=\beta^{2}=\beta=1$.

As observed from Figure 1, the threshold signal function $x^{1 *}$ is composed of two parts; one part that coincides with the 45 degree line, where the speculator is indifferent between attacking currency 1 and 2, and a flatter part, where the speculator is indifferent between attacking currency 1 and not attacking. Note that $x^{1 *}\left(x_{i}^{2}\right)<x_{s}^{*}$ and $x^{1 *}\left(x_{i}^{2}\right) \rightarrow x_{s}^{*}$ as $x_{i}^{2} \rightarrow \infty$, where $x_{s}^{*}$ is the threshold signal in the single-target case, given by (9). This can be understood as follows. Since each speculator expects some other speculators to attack currency 2, the expected payoff from attacking currency 1 is, for any $x_{i}^{2}$, lower than with a single target. So, speculators choose a less aggressive attacking strategy, and thus $x^{1 *}\left(x_{i}^{2}\right)$ lies below $x_{s}^{*}$. But as $x_{i}^{2}$ increases, a speculator infers greater values of $\theta^{2}$ and expects a smaller fraction of other speculators to attack currency 2 , so the presence of currency 2 will have a smaller effect on the speculator's decision to attack currency 1. As $x_{i}^{2} \rightarrow \infty$, the presence of country 2 becomes irrelevant, and the speculator behaves as if currency 1 is the only target. Speculators' attacking decisions for given signals $\left(x_{i}^{1}, x_{i}^{2}\right)$ can be observed from Figure 2. Observe that functions $x^{1 *}$ and $x^{2 *}$ divide the $\left(x_{i}^{1}, x_{i}^{2}\right)$ space into three regions according to the corresponding attacking decision.

Similarly, Figure 3 indicates that $\theta^{1 *}\left(\theta^{2}\right)<\theta_{s}^{*}$ and $\theta^{1 *}\left(\theta^{2}\right) \rightarrow \theta_{s}^{*}$ as $\theta^{2} \rightarrow \infty$, where $\theta_{s}^{*}$ is the threshold fundamentals in the single-target case, given by (8). Thus, the presence of a second target always makes the survival of a peg more likely. This implication is not surprising because, given that the total measure of speculators is fixed, the presence of multiple targets serves to diversify the attacking pressure. Of greater importance is that $\theta^{1 *}\left(\theta^{2}\right)$ is highly dependent on the value of $\theta^{2}$. This can be more clearly observed in Figure 4 , in which $\theta^{1 *}$ and $\theta^{2 *}$ divide the $\left(\theta^{1}, \theta^{2}\right)$ space into four regions according to the outcomes of attack for the two countries. Figure 4 shows that the success of attack on one country depends critically on the other country's fundamentals, and not just on its own. Put differently, what matters here is not necessarily the value of the fundamentals per se, but their value relative to the other target. Such a feature is completely lacking in the public information case discussed in Section 2.2.1, unless it is exogenously imposed through an equilibrium selection device. ${ }^{18}$

\footnotetext{
${ }^{18}$ For example, one can assume that in the multiple-equilibria region in which at most one peg can fail $\left(\theta^{1}, \theta^{2} \in(0,1]\right.$ and $\left.\theta^{1}+\theta^{2}>1\right)$, the probability of speculators coordinating to attack currency 1 is increasing in $\theta^{2}$, and vice versa. While such assumption may sound plausible, it is totally ad hoc.
} 
The comparison between $\theta^{1 *}$ and $\theta_{s}^{*}$ above assumes the same total measure of speculators, normalized to one, for single and two-country environments. One may ask what happens if the per country, not total, measure of speculators is fixed. In other words, the question is whether doubling both the number of targets and the population of speculators, or equivalently, pooling the speculators who were segmented into two distinct markets, makes collapse of the peg more likely or less so. This is an interesting question because, while doubling the population of speculators increases the potential size of the attack towards each country, the presence of multiple targets may make coordination among speculators more difficult, and it is not obvious which of the two channels dominates. To answer this question, Figure 5 draws $\theta^{1 *}$ and $\theta^{2 *}$ along with the lines corresponding to $\theta_{s, 0.5}^{*}$, the threshold value of fundamentals in the single-target case with measure 0.5 of speculators. ${ }^{19}$ Note that $\theta^{1 *}$ can be lower than $\theta_{s, 0.5}^{*}$, which implies that currency 1 may be able to sustain the peg, even with the values of fundamentals that force country 1 to abandon the peg in the single-target case with half the population of speculators. However, this can occur only when country 2 falls victim to attack; indeed, the region of $\left(\theta^{1}, \theta^{2}\right)$, under which both currencies sustain the peg (i.e., the upper right region of Figure 5 in which both $\theta^{1}$ and $\theta^{2}$ are above the relevant threshold), is smaller when speculators are pooled. This finding turns out to be robust to changes in $\beta$ and $c$.

Another interesting exercise involves varying $\beta$. Figure 6 illustrates the outcomes of attack when $\beta^{1}$ and $\beta^{2}$ are raised to 4 , corresponding to more precise private signals than in Figure 4. Note from (8) that the value of $\theta_{s}^{*}$ is independent of $\beta$, hence Figures 4 and 6 are directly comparable. Comparing Figures 4 and 6 , we observe that in Figure $6, \theta^{1 *}$ is higher for relatively large values of $\theta^{2}$, and is lower for relatively small values of $\theta^{2}$. As a result, the region of fundamentals $\left(\theta^{1}, \theta^{2}\right)$ for which both pegs survive, and the region for which both pegs fail, are both smaller in Figure 6. The implication is that increased precision of signals has a decoupling effect, in the sense that the two countries are less likely to face the same outcomes of attack. When private signals become more precise, speculators are able to better discern the country with relatively weak fundamentals, hence the attacking pressure tends to be more concentrated on that country. Thus, the country with relatively strong fundamentals manages to survive the attack for a wider range of its own fundamentals, at the cost of placing the other country in a more vulnerable situation.

Conversely, if one interprets the case of simultaneous collapses of the peg as contagion, the model suggests that contagion is more likely with less precise signals. However, such an implication for contagion based on a one-shot game requires some caution, since the implication may be quite different if there are multiple rounds of speculative attacks.

\footnotetext{
${ }^{19}$ Proceeding as in Section 2.2.2, with the condition (7) modified as $\theta_{s, 0.5}^{*}=0.5 \Phi\left(\sqrt{\beta}\left(x_{s, 0.5}^{*}-\theta_{s, 0.5}^{*}\right)\right)$, one obtains $\theta_{s, 0.5}^{*}=0.5(1-c)=0.5 \theta_{s}^{*}$, and $x_{s, 0.5}^{*}=\theta_{s, 0.5}^{*}+\frac{1}{\sqrt{\beta}} \Phi^{-1}\left(2 \theta_{s, 0.5}^{*}\right)$.
} 
To see this, note that greater precision of signals expands the region of fundamentals $\left(\theta^{1}, \theta^{2}\right)$ for which at least one country abandons the peg. But once one country floats its currency, the remaining country faces all speculators alone, such that it must now abandon the peg for a wider range of its own fundamentals. If contagion is interpreted in such an environment under repeated speculative attacks, then more accurate information indeed facilitates contagion.

\subsubsection{Nonsymmetric Targets}

Let us now examine the nonsymmetric case, where $c^{1}=c^{2}=0.1, \beta^{1}=1$, and $\beta^{2}=4$. Figure 7 shows the attacking decisions in the $\left(x_{i}^{1}, x_{i}^{2}\right)$ space. Note that unlike in Figure 2, the part of $x^{1 *}\left(x_{i}^{2}\right)$ where the speculator is indifferent between attacking currency 1 and 2 is steeper than the 45 degree line. The intuition is that country 2's signal, which is more precise, has a stronger impact on the speculator's decision than country 1's signal. For example, when both $x_{i}^{1}$ and $x_{i}^{2}$ are relatively large (say, equal to 1 ), speculators recognize that $\theta^{2}$ is more certain to be strong than $\theta^{1}$, and hence prefer to attack currency 1 over currency 2 . The opposite is true when both $x_{i}^{1}$ and $x_{i}^{2}$ are relatively small.

Figure 8 illustrates the outcomes of attack in the $\left(\theta^{1}, \theta^{2}\right)$ space. Note that the outcome of attack for currency 1 is more strongly dependent on $\theta^{2}$ than the outcome of attack for currency 2 is on $\theta^{1}$, which can be understood as follows. Since $\beta^{1}<\beta^{2}$, country 2's fundamentals, whether strong or weak, are perceived more accurately by speculators. Thus, when country 2 has strong fundamentals, speculators recognize this and tend to shift their target to country 1, placing country 1 in a vulnerable position. Conversely, when country 2 has weak fundamentals, speculators also recognize this, and tend to shift their target to country 2, relaxing the attacking pressure on country 1 .

This explanation suggests that what really matters for the profile of the threshold fundamentals function of a country (say, $\theta^{1 *}$ ) is the precision of the signal for the other country $\left(\beta^{2}\right)$, not its own $\left(\beta^{1}\right)$. Indeed, we observe that in Figure $8, \theta^{1 *}$ resembles that in Figure 6 , where $\beta^{1}=\beta^{2}=4$, whereas $\theta^{2 *}$ is similar to that in Figure 4 , where $\beta^{1}=\beta^{2}=1$.

The implication of this observation, with respect to the issues of contagion and decoupling mentioned above, is as follows. Suppose the precision of private signals reflects the transparency of government policy ${ }^{20}$, such that country $j$ 's government authority can, to some extent, control $\beta^{j}$. Then, what country 1's government authority can affect is mainly how the outcome of attack for currency 2 depends on country 1's fundamentals; the issue which is probably more important to country 1's government authority, the de-

\footnotetext{
${ }^{20}$ Heinemann and Illing (2002) adopts such an interpretation and discusses the impact of transparency on the probability of successful speculative attack.
} 
pendence of the outcome of attack for currency 1 on country 2's fundamentals, is instead at the discretion of country 2's government authority.

\subsection{Symmetric $N=3$ Targets}

I conclude this section by setting forth an example for the symmetric $N>2$ case. Figures 9 and 10 depict the equilibrium threshold functions for $N=3$, where $c^{j}=0.1$ and $\beta^{j}=1$ for all $j$. Note that the threshold functions are symmetric across $j$.

Figure 9 depicts $x^{3 *}\left(x_{i}^{1}, x_{i}^{2}\right)$; speculators receiving signals below the graph attack country 3. As in the case of $N=2, x^{3 *}\left(x_{i}^{1}, x_{i}^{2}\right)$ is composed of two parts; the part that coincides with $\min \left\{x_{i}^{1}, x_{i}^{2}\right\}$, where the speculator is indifferent between attacking currency 3 and one of the other two currencies, and the flatter part, where the speculator is indifferent between attacking currency 3 and not attacking. While $x_{s}^{*}$ is not indicated in the figure to avoid graphical clutter, $x^{3 *}\left(x_{i}^{1}, x_{i}^{2}\right)<x_{s}^{*}$ and $x^{3 *}$ approaches $x_{s}^{*}$ as both $x_{i}^{1}$ and $x_{i}^{2}$ tend to $\infty$. The intuition is similar to the $N=2$ case. Compared to the single-target case, speculators choose a less aggressive attacking strategy towards country 3 , since the attacking pressure is spread out over three countries. But as $x_{i}^{1}$ and $x_{i}^{2}$ increase, speculators expect a smaller fraction of other speculators to attack these countries, so the presence of the other two countries becomes less important for the decision to attack country 3 . As both $x_{i}^{1}$ and $x_{i}^{2}$ approach $\infty$, the speculator behaves as if currency 3 is the only target. Note that $x^{1 *}, x^{2 *}$ and $x^{3 *}$ divide the $\left(x_{i}^{1}, x_{i}^{2}, x_{i}^{3}\right)$ space into four regions according to speculators' attacking decisions.

Figure 10 illustrates $\theta^{3 *}\left(\theta^{1}, \theta^{2}\right)$; country 3 abandons the peg when the combination of the fundamentals lies on or below the graph. As observed from Figure 10, $\theta^{3 *}\left(\theta^{1}, \theta^{2}\right)$ increases smoothly in $\theta^{1}$ and $\theta^{2}$. Moreover, $\theta^{3 *}\left(\theta^{1}, \theta^{2}\right)$ approaches 0 as either $\theta^{1}$ or $\theta^{2}$ tends to $-\infty$, and approaches $\theta_{s}^{*}$ as both $\theta^{1}$ and $\theta^{2}$ approach $\infty$. This implies that if there is one country with very weak fundamentals, speculators target this country, relaxing the attacking pressure on other countries. The threshold fundamentals functions for the three countries divide the $\left(\theta^{1}, \theta^{2}, \theta^{3}\right)$ space into eight regions according to the outcomes of attack for the three countries.

\section{Discussions and Extensions}

\subsection{Introduction of Public Information}

I have thus far restricted speculators' information on the fundamentals to private signals by assuming an uninformative prior of the fundamentals. Here, I relax this 
assumption and introduce public information.

In the standard model with a single target, it has been shown (e.g., Morris and Shin (2002), Hellwig (2002)) that multiple equilibria may exist if there is public information that is sufficiently informative relative to private information. For example, when the fundamentals $\theta$ are drawn from a common prior $N(y, 1 / \alpha)$ in the model of Section 2.2.2, then there may be multiple equilibria if $\alpha / \sqrt{\beta}>\sqrt{2 \pi}$. In other words, the sufficient condition for a unique equilibrium is $\alpha / \sqrt{\beta} \leq \sqrt{2 \pi}$. The following proposition provides the corresponding sufficient condition in the model with multiple targets.

Proposition 4. (1) For $N=2$, suppose the common prior distribution of $\theta^{j}$ is $N\left(y^{j}, 1 / \alpha^{j}\right)$ for $j=\{1,2\}$. Proposition 1 holds if $\alpha^{j} / \sqrt{\beta^{j}}<\sqrt{2 \pi}$ for $j=\{1,2\}$. (2) For any $N$, suppose that $\beta^{j}=\beta>0, c^{j}=c \in(0,1)$, and that the common prior distribution of $\theta^{j}$ is $N(y, 1 / \alpha)$ for all $j \in\{1,2, \ldots, N\}$. Proposition 3 holds if $\alpha / \sqrt{\beta}<\sqrt{2 \pi}$.

When the sufficient condition in Proposition 4 is not satisfied, it turns out that the corresponding operator cannot be proved to be a contraction. Note that the sufficient condition for a unique combination of equilibrium threshold functions is very similar to the sufficient condition for a unique equilibrium in the single-target case. Such similarity is not a coincidence. In fact, it is possible to apply the contraction approach used in this paper to the single-target environment, as can be seen as follows.

Consider a $N=2$ case with $c^{2}>1$, such that while there are two potential targets, attacking currency 2 is never profitable. Then, no speculator ever attacks currency 2, hence $\underline{\theta}_{n}^{1 *}\left(\theta^{2}\right)$ and $\bar{\theta}_{n}^{1 *}\left(\theta^{2}\right)$ become independent of $\theta^{2}$. Using such constant valued functions, we can proceed with the iterative deletion as in the proof of Proposition 4(1); then, under the condition $\alpha^{1} / \sqrt{\beta^{1}}<\sqrt{2 \pi}$ which guarantees that $T$ is a contraction, $\underline{\theta}_{n}^{1 *}$ and $\bar{\theta}_{n}^{1 *}$ converge to the same, constant valued function, which establishes the unique equilibrium result. Except for the inequality being strict, this condition coincides with the known sufficient condition for equilibrium uniqueness. ${ }^{21}$

\subsection{Correlated Noises}

This paper has assumed throughout that the noises $\epsilon_{i}^{j}$ are independent across $j$. This assumption enables the focus on the direct impact of increasing the number of targets and facilitates comparison with the two benchmark models; however, it is not crucial for

\footnotetext{
${ }^{21}$ When $\alpha^{1} / \sqrt{\beta^{1}}=\sqrt{2 \pi}$, the operator $T$ is only a weak contraction, hence the contraction mapping theorem does not apply. However, in the single-target case, a slight reformulation yields an operator which maps a compact space to itself, so one can instead invoke Edelstein's fixed point theorem (Edelstein (1962)) to show that there exists a unique fixed point, and thus a unique equilibrium.
} 
the existence and uniqueness of equilibrium threshold functions shown in Section 3, as stated by the proposition below.

Proposition 5. (1) For $N=2$, suppose $\epsilon_{i}^{j}, j=\{1,2\}$, are drawn from a continuous and bounded joint pdf $\varphi: \mathbb{R}^{2} \rightarrow \mathbb{R}^{++}$. Then, Proposition 1 holds. (2) For $N>2$, suppose $\beta^{j}=\beta>0$ and $c^{j}=c \in(0,1)$ for all $j$, and suppose $\epsilon_{i}^{j}, j=\{1,2, \ldots, N\}$, are drawn from a continuous bounded joint pdf $\varphi: \mathbb{R}^{N} \rightarrow \mathbb{R}^{++}$that is symmetric across $j$. Then, Proposition 3 holds.

At first glance, correlated noises may appear to create complications in establishing that the relevant operator is a contraction. Proposition 5 states, however, that this is not the case, and the same equilibrium properties can be obtained even when noise is correlated. Intuitively, this is because, whatever the correlation among the noises, a higher value of $x_{i}^{j}$ suggests a higher value of $\theta^{j}$, and a higher value of $\theta^{j}$ increases the proportion of strong signals $x_{i}^{j}$, which is key for the equilibrium to be characterized by threshold functions.

\section{Conclusions}

This paper has examined a global games model of speculative attacks in which there exist multiple targets for speculators to attack. With two countries, or any $N$ symmetric countries, the equilibrium is characterized by threshold signal functions and threshold fundamentals functions, which are uniquely determined for each country. Accordingly, in these cases, a country's fundamentals are evaluated in relation to those of other countries, and the outcomes of attack are uniquely determined given the fundamentals of all countries. Such an equilibrium property is a natural extension to that in the canonical global games model with a single target, and it starkly contrasts with that in a multiple-target environment with publicly observed fundamentals.

This paper has set forth a number of numerical exercises to generate further insight into these equilibrium threshold functions, mainly focusing on the two-country case, and has derived several implications. First, the addition of the second target lowers the threshold fundamentals and facilitates a country to sustain the peg, if the total population of speculators is held constant. When instead the per-country population of speculators is held constant, the threshold fundamentals may still be lower than in the single-target case, but only when the other country falls victim to speculative attack. Second, increased quality of private information has a decoupling effect, such that under greater precision of signals, the two countries are less likely to face the same economic outcome. This is because, with greater precision of signals, speculators are more capable of uncovering 
the country with the weaker fundamentals and of coordinating their attacks on that country. Third, the precision of signals for country 1 mainly affects how country 2's threshold fundamentals function varies with country 1's fundamentals, not how country 1 's threshold fundamentals function varies with country 2's fundamentals. This restricts the usefulness, as a policy tool, of varying the transparency of government policy, unless countries coordinate on such policy.

Finally, this paper has considered the extension of introducing public information through an informative prior of the fundamentals, and has shown that the sufficient condition for the existence of unique equilibrium threshold functions closely resembles the known sufficient condition for the unique equilibrium in the single-target case. In order to relate these conditions, this paper has described how its contraction argument can be applied to the single-target case.

The environment of this paper, namely global games of regime change with multiple regimes, is not only of theoretical interest, but has many interesting real-life applications. Combining the iterative deletion procedure with the contraction mapping argument turns out to be a powerful approach for examining such an environment, not only for the theoretical analysis of the equilibrium, but also for numerical computations. One limitation of this approach is that it is not applicable when there are more than two nonsymmetric targets, in which case the threshold functions may not always be nondecreasing. Obtaining, for example, sufficient conditions for unique threshold functions in such an environment appears to require a different approach, and such pursuit is left for future research. 


\section{Appendix A: Proofs}

\section{Proposition 1}

Let operator $T$ and spaces $B\left(X, \mathbb{R}^{2 N}\right), \tilde{B}\left(X, \mathbb{R}^{2 N}\right)$ be as defined in Section 3.3. If one can show that $\tilde{B}\left(\mathbb{R}^{N}, \mathbb{R}^{2 N}\right)$ is a complete metric space, that $T$ maps from $\tilde{B}\left(\mathbb{R}^{N}, \mathbb{R}^{2 N}\right)$ to itself, and that $T$ is a contraction, then the contraction mapping theorem implies that there exists a unique fixed point $g^{*}=\left(\underline{\theta}^{*}, \bar{\theta}^{*}\right)$ of $T$. The proof then concludes by establishing the properties of this fixed point, most importantly $\underline{\theta}^{*}=\bar{\theta}^{*}$. Let these steps be proved through a series of lemma.

Lemma 1. For any $X \subseteq \mathbb{R}^{l}, B\left(X, \mathbb{R}^{N}\right)$ is a Banach space.

Proof. That $B\left(X, \mathbb{R}^{N}\right)$ is a normed vector space easily follows from the definition of a normed vector space. It remains to show that $B\left(X, \mathbb{R}^{N}\right)$ is complete, so that if $\left\{g_{n}\right\}_{n=1}^{\infty}$, $g_{n} \in B\left(X, \mathbb{R}^{N}\right) \forall n$, is a Cauchy sequence, there exists $g \in B\left(X, \mathbb{R}^{N}\right)$ such that, for any $\epsilon>0$, there exists $M_{\epsilon}$ such that $\left\|g_{n}-g\right\| \leq \epsilon$, all $n \geq M_{\epsilon}$, where $\|\cdot\|$ is the max-sup norm. This is shown by extending the proof that the space of scalar-valued, bounded continuous functions is complete (see e.g., Stokey and Lucas (1989), Theorem 3.1), and involves three steps: (1) to find a candidate for $g$, (2) to show $\left\{g_{n}\right\}$ converges to $g$ in the max-sup norm, and (3) to show $g \in B\left(X, \mathbb{R}^{N}\right)$. First, fix any $z \in X$. Then the sequence of real numbers $\left\{g_{n}^{j}(z)\right\}$ satisfies

$$
\left|g_{n}^{j}(z)-g_{m}^{j}(z)\right| \leq \sup _{w \in X}\left|g_{n}^{j}(w)-g_{m}^{j}(w)\right| \leq \max _{j} \sup _{w \in X}\left|g_{n}^{j}(w)-g_{m}^{j}(w)\right|=|| g_{n}-g_{m} \|,
$$

so it satisfies a Cauchy criterion, since $\left\{g_{n}\right\}$ is a Cauchy sequence by assumption, and its convergence to some $g^{j}(z) \in \mathbb{R}$ is assured since the space of real numbers is complete. So the candidate for $g$ is $g=\left(g^{1}, g^{2}, \ldots, g^{N}\right)$, where $g_{n}^{j} \rightarrow g^{j}$ pointwise for each $j$. Next, take any $\epsilon>0$ and choose $M_{\epsilon}$ so that $n, m \geq M_{\epsilon}$ implies $\left\|g_{n}-g_{m}\right\| \leq \epsilon / 2$. This is possible since $\left\{g_{n}\right\}$ is a Cauchy sequence. Then, for any $z \in X$ and $m \geq n \geq M_{\epsilon}$,

$$
\begin{aligned}
\left|g_{n}^{j}(z)-g^{j}(z)\right| & \leq\left|g_{n}^{j}(z)-g_{m}^{j}(z)\right|+\left|g_{m}^{j}(z)-g^{j}(z)\right| \\
& \leq|| g_{n}-g_{m}||+\left|g_{m}^{j}(z)-g^{j}(z)\right| \\
& \leq \epsilon / 2+\left|g_{m}^{j}(z)-g^{j}(z)\right| .
\end{aligned}
$$

Since $g_{n}^{j} \rightarrow g^{j}$ pointwise, $m$ can be chosen separately for each $z$ so that $\left|g_{m}^{j}(z)-g^{j}(z)\right| \leq$ $\epsilon / 2$. Since the choice of $z \in X$ was arbitrary, it follows that $\sup _{z \in X}\left|g_{n}^{j}(z)-g^{j}(z)\right| \leq \epsilon$, all $n \geq M_{\epsilon}$. Since this holds for any $j$, it follows that $\left\|g_{n}-g\right\| \leq \epsilon$, all $n \geq M_{\epsilon}$. Thus $\left\|g_{n}-g\right\| \rightarrow 0$ as $n \rightarrow \infty$, since the choice of $\epsilon$ was arbitrary. Finally, let us show that $g \in B\left(X, \mathbb{R}^{N}\right)$, which is true if each $g^{j}$ is bounded, continuous and nondecreasing. 
Boundedness is obvious. For continuity, one needs to show that for any $\epsilon>0$ and any $z \in X$, there exists $\delta>0$ such that $\left|g^{j}(z)-g^{j}(w)\right|<\epsilon$ if $\|z-w\|_{E}<\delta$, where $\|\cdot\|_{E}$ is the Euclidean norm on $\mathbb{R}^{l}$. For any $j$, take any $\epsilon, z$ and choose $k$ so that $\sup _{w \in X}\left|g^{j}(w)-g_{k}^{j}(w)\right|<\epsilon / 3$. This is possible because, since $g_{n} \rightarrow g$ in the max-sup norm, each $g_{n}^{j} \rightarrow g^{j}$ in the sup norm. Then choose $\delta$ such that $\|z-w\|_{E}<\delta$ implies $\left|g_{k}^{j}(z)-g_{k}^{j}(w)\right|<\epsilon / 3$. Since $g_{k}^{j}$ is continuous, such a choice is possible. Then

$$
\begin{aligned}
\left|g^{j}(z)-g^{j}(w)\right| & \leq\left|g^{j}(z)-g_{k}^{j}(z)\right|+\left|g_{k}^{j}(z)-g_{k}^{j}(w)\right|+\left|g_{k}^{j}(w)-g^{j}(w)\right| \\
& \leq 2 \sup _{w \in X}\left|g^{j}(w)-g_{k}^{j}(w)\right|+\left|g_{k}^{j}(z)-g_{k}^{j}(w)\right| \\
& <\epsilon
\end{aligned}
$$

so $g^{j}$ is continuous for all $j$. Therefore $g \in B\left(X, \mathbb{R}^{N}\right)$, which completes the proof.

Given Lemma $1, B\left(X, \mathbb{R}^{N}\right)$ is a complete metric space with the metric $d(f, g)=\|f-g\|$ for $f, g \in B\left(X, \mathbb{R}^{N}\right)$, where $\|\cdot\|$ is the max-sup norm. Thus, being a closed subset of $B\left(X, \mathbb{R}^{N}\right), \tilde{B}\left(X, \mathbb{R}^{N}\right)$ is also a complete metric space with the max-sup norm. Since this is true for any $X \subseteq \mathbb{R}^{l}$ and $N, \tilde{B}\left(\mathbb{R}^{2}, \mathbb{R}^{4}\right)$ is a complete metric space.

Lemma 2. $T$ maps $\tilde{B}\left(\mathbb{R}^{2}, \mathbb{R}^{4}\right)$ to itself.

Proof. Take any $g_{n}^{*}$ such that $g_{n}^{*}(\theta)=\left(\underline{\theta}_{n}^{1 *}\left(\theta^{2}\right), \underline{\theta}_{n}^{2 *}\left(\theta^{1}\right), \bar{\theta}_{n}^{1 *}\left(\theta^{2}\right), \bar{\theta}_{n}^{2 *}\left(\theta^{1}\right)\right) \in \tilde{B}\left(\mathbb{R}^{2}, \mathbb{R}^{4}\right)$. To prove $g_{n+1}^{*}=T g_{n}^{*} \in \tilde{B}\left(\mathbb{R}^{2}, \mathbb{R}^{4}\right)$, one must show that all component functions of $g_{n+1}^{*}$, namely $\underline{\theta}_{n+1}^{j *}$ and $\bar{\theta}_{n+1}^{j *}, j=\{1,2\}$, are bounded, continuous and nondecreasing. Boundedness is obvious since $\underline{\theta}_{n+1}^{j *}, \bar{\theta}_{n+1}^{j *} \in(0,1)$ from $(21)$ and $(22)$. For continuity, note from (17) and (18) that if $\underline{\theta}_{n}^{j *}$ and $\bar{\theta}_{n}^{j *}$ are bounded, continuous and nondecreasing for $j=\{1,2\}$, then $\underline{\Gamma}_{n}^{j *}\left(x_{i}\right)-\bar{\Gamma}_{n}^{k *}\left(x_{i}\right)$ and $\bar{\Gamma}_{n}^{j *}\left(x_{i}\right)-\underline{\Gamma}_{n}^{k *}\left(x_{i}\right)$ are bounded and continuous in both arguments, decreasing in $x_{i}^{j}$, and nondecreasing in $x_{i}^{k}$. Then, (19) and (20) imply that $\underline{x}_{n}^{j *}\left(x_{i}^{k}\right)$ and $\bar{x}_{n}^{j *}\left(x_{i}^{k}\right)$ are continuous and nondecreasing, and increasing for the range of $x_{i}^{k}$ for which $\underline{x}_{n}^{j *}\left(x_{i}^{k}\right)$ and $\bar{x}_{n}^{j *}\left(x_{i}^{k}\right)$ are determined by the indifference condition between attacking $j$ and $k$ under the corresponding beliefs. It then follows from (21) and (22) that $\underline{\theta}_{n+1}^{j}$ and $\bar{\theta}_{n+1}^{j}$ are continuous and nondecreasing (in fact, increasing), hence $g_{n+1}^{*} \in$ $\tilde{B}\left(\mathbb{R}^{2}, \mathbb{R}^{4}\right)$.

Lemma 3. $T$ is a contraction.

Proof. The proof proceeds by showing that the Blackwell's sufficient conditions for a contraction are satisfied for each component function. Take any $g_{n}^{*}(\theta)=\left(\underline{\theta}_{n}^{1 *}\left(\theta^{2}\right), \underline{\theta}_{n}^{2 *}\left(\theta^{1}\right), \bar{\theta}_{n}^{1 *}\left(\theta^{2}\right), \bar{\theta}_{n}^{2 *}\left(\theta^{1}\right.\right.$ $\tilde{B}\left(\mathbb{R}^{2}, \mathbb{R}^{4}\right)$. For monotonicity, fix $j \in\{1,2\}$ and define $g_{n}^{* *} \in \tilde{B}\left(\mathbb{R}^{2}, \mathbb{R}^{4}\right)$ by replacing the $j$-th component of $g_{n}^{*}$, namely $\underline{\theta}_{n}^{j *}$, by $\underline{\theta}_{n}^{j * *} \geq \underline{\theta}_{n}^{j *}$. Further, let $\underline{\Gamma}_{n}^{* *}, \bar{\Gamma}_{n}^{* *}, \underline{x}_{n}^{* *}, \bar{x}_{n}^{* *}, \underline{\theta}_{n+1}^{* *}$, 
$\bar{\theta}_{n+1}^{* *}$ be the functions obtained from $g_{n}^{* *}$ by $(17)-(22)$. For any $x_{i}=\left(x_{i}^{j}, x_{i}^{-j}\right) \in \mathbb{R}^{2}$, and (18) imply $\underline{\Gamma}_{n}^{j * *}\left(x_{i}\right) \geq \underline{\Gamma}_{n}^{j *}\left(x_{i}\right)$, whereas $\bar{\Gamma}_{n}^{* *}\left(x_{i}\right)=\bar{\Gamma}_{n}^{*}\left(x_{i}\right)$. Then, since $\underline{\Gamma}_{n}^{j * *}\left(x_{i}\right)$ and $\underline{\Gamma}_{n}^{j *}\left(x_{i}\right)$ are decreasing in $x_{i}^{j}$, whereas $\bar{\Gamma}_{n}^{k * *}\left(x_{i}\right)$ and $\bar{\Gamma}_{n}^{k *}\left(x_{i}\right)$ are nondecreasing in $x_{i}^{j}$ for $k \neq j$, (19) implies $\underline{x}_{n}^{j * *} \geq \underline{x}_{n}^{j *}$. Thus, (21) implies $\underline{\theta}_{n+1}^{j * *} \geq \underline{\theta}_{n+1}^{j *}$, hence monotonicity holds. For discounting, fix $j \in\{1,2\}$, take any $a \geq 0$ and define $g_{n}^{\#} \in \tilde{B}\left(\mathbb{R}^{2}, \mathbb{R}^{4}\right)$ by replacing the $j$-th component of $g_{n}^{*}$, namely $\underline{\theta}_{n}^{j *}$, by $\underline{\theta}_{n}^{j *}+a$. Let $\underline{\Gamma}_{n}^{\#}, \bar{\Gamma}_{n}^{\#}, \underline{x}_{n}^{\#}, \bar{x}_{n}^{\#}, \underline{\theta}_{n+1}^{\#}, \bar{\theta}_{n+1}^{\#}$ be the functions obtained from $g_{n}^{\#}$ by $(17)-(22)$. Then, noting that $\underline{\Gamma}_{n}^{j \#}\left(x_{i}\right)$ and $\underline{\Gamma}_{n}^{j *}\left(x_{i}\right)$ are decreasing in $x_{i}^{j}$, whereas $\bar{\Gamma}_{n}^{k \#}\left(x_{i}\right)$ and $\bar{\Gamma}_{n}^{k *}\left(x_{i}\right)$ are nondecreasing in $x_{i}^{j}$ for $k \neq j$, $\underline{x}_{n}^{j \#} \leq \underline{x}_{n}^{j *}+a$ follows from (17)-(20). Now, define $\underline{\theta}_{n+1}^{j \# \#}\left(\theta^{-j}\right)$ by

$$
\begin{aligned}
& \underline{\theta}_{n+1}^{j \# \#}\left(\theta^{-j}\right) \\
& =\int_{\epsilon_{i}^{-j} \in \mathbb{R}^{N-1}} \tilde{\phi}^{-j}\left(\epsilon_{i}^{-j}\right) \Phi\left(\sqrt{\beta^{j}}\left(\underline{x}_{n}^{j *}\left(\theta^{-j}+\epsilon_{i}^{-j}\right)+a-\underline{\theta}_{n+1}^{j \# \#}\left(\theta^{-j}\right)\right)\right) d \epsilon_{i}^{-j} .
\end{aligned}
$$

Since $\underline{x}_{n}^{j \#} \leq \underline{x}_{n}^{j *}+a, \underline{\theta}_{n+1}^{j \#} \leq \underline{\theta}_{n+1}^{j \# \#}$. Let $\gamma^{j}$ be the maximum value of the slope of $\Phi\left(\sqrt{\beta^{j}} z\right)$ for $z \in \mathbb{R}$, that is, $\gamma^{j}=\sqrt{\beta^{j}} \phi(0)=\sqrt{\beta^{j}} / \sqrt{2 \pi}>0$. Then, $\Phi\left(\sqrt{\beta^{j}}(z+a)\right)-\Phi\left(\sqrt{\beta^{j}} z\right) \leq$ $\gamma^{j} a$ for any $a \geq 0$. Thus, from (21) and (33),

$$
\begin{aligned}
\underline{\theta}_{n+1}^{j \# \#}\left(\theta^{-j}\right)-\underline{\theta}_{n+1}^{j *}\left(\theta^{-j}\right) & \leq \int_{\epsilon_{i}^{-j} \in \mathbb{R}^{N-1}} \tilde{\phi}^{-j}\left(\epsilon_{i}^{-j}\right) \gamma^{j}\left[a-\left(\underline{\theta}_{n+1}^{j \# \#}\left(\theta^{-j}\right)-\underline{\theta}_{n+1}^{j *}\left(\theta^{-j}\right)\right)\right] d \epsilon_{i}^{-j} \\
& \leq \gamma^{j}\left[a-\left(\underline{\theta}_{n+1}^{j \# \#}\left(\theta^{-j}\right)-\underline{\theta}_{n+1}^{j *}\left(\theta^{-j}\right)\right)\right] \\
& \leq \lambda^{j} a,
\end{aligned}
$$

where $\lambda^{j} \equiv \gamma^{j} /\left(1+\gamma^{j}\right) \in(0,1)$. Thus, $\underline{\theta}_{n+1}^{j \#}\left(\theta^{-j}\right) \leq \underline{\theta}_{n+1}^{j \# \#}\left(\theta^{-j}\right) \leq \underline{\theta}_{n+1}^{j *}\left(\theta^{-j}\right)+\lambda^{j} a$, and since the choice of $a \geq 0$ was arbitrary, discounting also holds. Repeating the argument above for each component function of $g_{n}^{*}$, it follows that monotonicity and discounting holds for $\underline{\theta}_{n}^{j *}$ and $\bar{\theta}_{n}^{j *}, j=\{1,2\}$. Now, let $g_{n}^{*}$ and $g_{n+1}^{*}$ be as defined above, take any $g_{n}^{+} \in \tilde{B}\left(\mathbb{R}^{2}, \mathbb{R}^{4}\right)$ such that

$g_{n}^{+}(\theta)=\left(\underline{\theta}_{n}^{1+}\left(\theta^{2}\right), \underline{\theta}_{n}^{2+}\left(\theta^{1}\right), \bar{\theta}_{n}^{1+}\left(\theta^{2}\right), \bar{\theta}_{n}^{2+}\left(\theta^{1}\right)\right)$, and let $g_{n+1}^{+}=T g_{n}^{+}$. Then, noting

$$
\underline{\theta}_{n}^{j *} \leq \underline{\theta}_{n}^{j+}+\sup _{\theta^{-j} \in \mathbb{R}^{N-1}}\left|\underline{\theta}_{n}^{j *}\left(\theta^{-j}\right)-\underline{\theta}_{n}^{j+}\left(\theta^{-j}\right)\right|
$$

and invoking monotonicity and discounting shown above,

$$
\underline{\theta}_{n+1}^{j *} \leq \underline{\theta}_{n+1}^{j+}+\lambda^{j} \sup _{\theta^{-j} \in \mathbb{R}^{N-1}}\left|\underline{\theta}_{n}^{j *}\left(\theta^{-j}\right)-\underline{\theta}_{n}^{j+}\left(\theta^{-j}\right)\right| .
$$

Reversing the role of $\underline{\theta}_{n}^{j *}$ and $\underline{\theta}_{n}^{j+}$, one obtains

$$
\underline{\theta}_{n+1}^{j+} \leq \underline{\theta}_{n+1}^{j *}+\lambda^{j} \sup _{\theta^{-j} \in \mathbb{R}^{N-1}}\left|\underline{\theta}_{n}^{j *}\left(\theta^{-j}\right)-\underline{\theta}_{n}^{j+}\left(\theta^{-j}\right)\right| .
$$


Therefore,

$$
\sup _{\theta^{-j} \in \mathbb{R}^{N-1}}\left|\underline{\theta}_{n+1}^{j+}\left(\theta^{-j}\right)-\underline{\theta}_{n+1}^{j *}\left(\theta^{-j}\right)\right| \leq \lambda^{j} \sup _{\theta^{-j} \in \mathbb{R}^{N-1}}\left|\underline{\theta}_{n}^{j *}\left(\theta^{-j}\right)-\underline{\theta}_{n}^{j+}\left(\theta^{-j}\right)\right|
$$

for all $j$. A similar argument establishes

$$
\sup _{\theta^{-j} \in \mathbb{R}^{N-1}}\left|\bar{\theta}_{n+1}^{j+}\left(\theta^{-j}\right)-\bar{\theta}_{n+1}^{j *}\left(\theta^{-j}\right)\right| \leq \lambda^{j} \sup _{\theta^{-j} \in \mathbb{R}^{N-1}}\left|\bar{\theta}_{n}^{j *}\left(\theta^{-j}\right)-\bar{\theta}_{n}^{j+}\left(\theta^{-j}\right)\right|,
$$

for all $j$. But then, letting $\lambda \equiv \max _{j} \lambda^{j}$,

$$
\begin{aligned}
& \left\|T g_{n}^{*}-T g_{n}^{+}\right\| \\
& =\left\|g_{n+1}^{*}-g_{n+1}^{+}\right\| \\
& =\max _{j}\left[\max \left(\sup _{\theta^{-j} \in \mathbb{R}^{N-1}}\left|\underline{\theta}_{n+1}^{j *}\left(\theta^{-j}\right)-\underline{\theta}_{n+1}^{j+}\left(\theta^{-j}\right)\right|, \sup _{\theta^{-j} \in \mathbb{R}^{N-1}}\left|\bar{\theta}_{n+1}^{j *}\left(\theta^{-j}\right)-\bar{\theta}_{n+1}^{j+}\left(\theta^{-j}\right)\right|\right)\right] \\
& \leq \max _{j}\left[\lambda^{j} \max \left(\sup _{\theta^{-j} \in \mathbb{R}^{N-1}}\left|\underline{\theta}_{n}^{j *}\left(\theta^{-j}\right)-\underline{\theta}_{n}^{j+}\left(\theta^{-j}\right)\right| \sup _{\theta^{-j} \in \mathbb{R}^{N-1}}\left|\bar{\theta}_{n}^{j *}\left(\theta^{-j}\right)-\bar{\theta}_{n}^{j+}\left(\theta^{-j}\right)\right|\right)\right] \\
& \leq \lambda \max _{j}\left[\max \left(\sup _{\theta^{-j} \in \mathbb{R}^{N-1}}\left|\underline{\theta}_{n}^{j *}\left(\theta^{-j}\right)-\underline{\theta}_{n}^{j+}\left(\theta^{-j}\right)\right| \sup _{\theta^{-j} \in \mathbb{R}^{N-1}}\left|\bar{\theta}_{n}^{j *}\left(\theta^{-j}\right)-\bar{\theta}_{n}^{j+}\left(\theta^{-j}\right)\right|\right)\right] \\
& =\lambda\left\|g_{n}^{*}-g_{n}^{+}\right\|,
\end{aligned}
$$

which implies that $T$ is a contraction.

Given Lemma 1-3, $T$ has a unique fixed point $g^{*}=\left(\underline{\theta}^{*}, \bar{\theta}^{*}\right)$ from the contraction mapping theorem. The final step of the proof is to show $\underline{\theta}^{*}=\bar{\theta}^{*} \equiv \theta^{*}$, where $\theta^{j *} \in(0,1)$ and $\theta^{j *}$ is continuous and increasing. Take $g_{n}^{*}(\theta)=\left(\underline{\theta}_{n}^{1 *}\left(\theta^{2}\right), \underline{\theta}_{n}^{2 *}\left(\theta^{1}\right), \bar{\theta}_{n}^{1 *}\left(\theta^{2}\right), \bar{\theta}_{n}^{2 *}\left(\theta^{1}\right)\right) \in$ $\tilde{B}\left(\mathbb{R}^{2}, \mathbb{R}^{4}\right)$ such that $\underline{\theta}_{n}^{j *}=\bar{\theta}_{n}^{j *}, j=\{1,2\}$. Then, (17) and (18) imply $\underline{\Gamma}_{n}^{j *}=\bar{\Gamma}_{n}^{j *} \equiv \Gamma_{n}^{j *}$. But then, (19) and (20) imply $\underline{x}_{n}^{j *}=\bar{x}_{n}^{j *}$. This is because, since $\Gamma_{n}^{j *}$ and $\Gamma_{n}^{j *}-\Gamma_{n}^{k *}$ are continuous and decreasing in $x_{i}^{j}$ for $j, k \in\{1,2\}, j \neq k$, either the set $\left\{x_{i}^{j} \mid \Gamma_{n}^{j *}\left(x_{i}\right) \geq\right.$ $\left.\max \left\{0,\left(\Gamma_{n}^{k *}\left(x_{i}\right)_{k \neq j}\right)\right\}\right\}$ is empty (in which case, $\underline{x}_{n}^{j *}=\bar{x}_{n}^{j *}=-\infty$ ), or else there is a single value of $x_{i}^{j}$ at which $\Gamma_{n}^{j *}\left(x_{i}\right)-\max \left\{0,\left(\Gamma_{n}^{k *}\left(x_{i}\right)\right)_{k \neq j}\right\}$ changes its sign. Therefore, (21) and (22) imply $\underline{\theta}_{n+1}^{j *}=\bar{\theta}_{n+1}^{j *} \equiv \theta_{n+1}^{j *}$. Moreover, arguing as in the proof of Lemma 2, $\theta_{n+1}^{j *} \in(0,1)$ and $\theta_{n+1}^{j *}$ is continuous and increasing.

Now, let $\tilde{B}^{\prime}\left(\mathbb{R}^{2}, \mathbb{R}^{4}\right)$ denote the space of bounded, continuous, nondecreasing vectorvalued functions $g: \mathbb{R}^{2} \rightarrow \mathbb{R}^{4}, g=\left(g^{1}, g^{2}, g^{3}, g^{4}\right)$ where $g^{1}=g^{3}$ and $g^{2}=g^{4}$, equipped with the max-sup norm $\|g\| \equiv \max _{j=1}^{N}\left(\sup _{z \in \mathbb{R}}\left|g^{j}(z)\right|\right)$. Further, let $\tilde{B}^{\prime \prime}\left(\mathbb{R}^{2}, \mathbb{R}^{4}\right) \subseteq \tilde{B}^{\prime}\left(\mathbb{R}^{2}, \mathbb{R}^{4}\right)$ be the space in which 'nondecreasing' in the definition of $\tilde{B}^{\prime}\left(\mathbb{R}^{2}, \mathbb{R}^{4}\right)$ is replaced by 'increasing', and $g^{j} \in(0,1)$ for all $j$. Then, $\tilde{B}^{\prime}\left(\mathbb{R}^{2}, \mathbb{R}^{4}\right)$ is a closed subset of $\tilde{B}\left(\mathbb{R}^{2}, \mathbb{R}^{4}\right)$, and the argument above implies $T\left(\tilde{B}^{\prime}\left(\mathbb{R}^{2}, \mathbb{R}^{4}\right)\right) \subseteq \tilde{B}^{\prime \prime}\left(\mathbb{R}^{2}, \mathbb{R}^{4}\right) \subseteq \tilde{B}\left(\mathbb{R}^{2}, \mathbb{R}^{4}\right)$, so it follows that $g^{*} \in \tilde{B}^{\prime \prime}\left(\mathbb{R}^{2}, \mathbb{R}^{4}\right)$ (Stokey and Lucas (1989), Theorem 3.2, Corollary 1). Therefore, $\underline{\theta}^{*}=\bar{\theta}^{*} \equiv \theta^{*}$, where $\theta^{j *} \in(0,1)$ and $\theta^{j *}$ is continuous and increasing, as was to be shown. 


\section{Proposition 2}

The proof is almost identical to the $N=2$ case. First, since $B\left(X, \mathbb{R}^{N}\right)$ is a Banach space as shown in Lemma $1, \tilde{B}\left(X, \mathbb{R}^{N}\right)$ is a complete metric space, and thus so is $\tilde{B}\left(\mathbb{R}^{N}, \mathbb{R}^{2 N}\right)$. That $T^{\prime}$ maps $\tilde{B}\left(\mathbb{R}^{N}, \mathbb{R}^{2 N}\right)$ to itself is also straightforward, except that this time, $\underline{x}_{n}^{j *}$ and $\bar{x}_{n}^{j *}$ may not always be nondecreasing, but $\underline{x}_{n, N D}^{j *}$ and $\bar{x}_{n, N D}^{j *}$ are nondecreasing by construction, such that $\underline{\theta}_{n+1}^{j}$ and $\bar{\theta}_{n+1}^{j}$ are nondecreasing (indeed, increasing). It remains only to confirm that using $\underline{x}_{n, N D}^{j *}$ and $\bar{x}_{n, N D}^{j *}$, instead of $\underline{x}_{n}^{j *}$ and $\bar{x}_{n}^{j *}$, to compute $\underline{\theta}_{n+1}^{j}$ and $\bar{\theta}_{n+1}^{j}$ does not hamper the satisfaction of the Blackwell's sufficient conditions for contraction.

For monotonicity, fix $j \in\{1,2, \ldots, N\}$ and define $g_{n}^{* *} \in \tilde{B}\left(\mathbb{R}^{N}, \mathbb{R}^{2 N}\right)$ by replacing the $j$-th component of $g_{n}^{*}$, namely $\underline{\theta}_{n}^{j *}$, by $\underline{\theta}_{n}^{j * *} \geq \underline{\theta}_{n}^{j *}$. Let $\underline{\Gamma}_{n}^{* *}, \bar{\Gamma}_{n}^{* *}, \underline{x}_{n}^{* *}, \bar{x}_{n}^{* *}, \underline{x}_{n, N D}^{* *}, \bar{x}_{n, N D}^{* *}, \underline{\theta}_{n+1}^{* *}$, $\bar{\theta}_{n+1}^{* *}$ be the functions obtained from $g_{n}^{* *}$ by $(17)-(20)$ and $(27)-(32)$, with $c^{j}=c$ and $\beta^{j}=\beta$ for all $j$. Proceeding as in the proof of Lemma 3, it follows that $\underline{x}_{n}^{j * *} \geq \underline{x}_{n}^{j *}$, and since $\underline{x}_{n}^{j *} \geq \underline{x}_{n, N D}^{j *}$ from the definition of $\underline{x}_{n, N D}^{j *}, \underline{x}_{n, N D}^{j *} \in \underline{X}_{n}^{j * *} \equiv\left\{f \in \tilde{B}\left(R^{N-1}, \mathbb{R}\right) \mid f \leq \underline{x}_{n}^{j * *}\right\}$. Then, $\underline{x}_{n, N D}^{j * *} \geq \underline{x}_{n, N D}^{j *}$ from the definition of $\underline{x}_{n, N D}^{j * *}$, and thus $\underline{\theta}_{n+1}^{j * *} \geq \underline{\theta}_{n+1}^{j *}$ from (31), which implies monotonicity.

For discounting, fix $j \in\{1,2, \ldots, N\}$, take any $a \geq 0$ and define $g_{n}^{\#} \in \tilde{B}\left(\mathbb{R}^{N}, \mathbb{R}^{2 N}\right)$ by replacing the $j$-th component of $g_{n}^{*}$, namely $\underline{\theta}_{n}^{j *}$, by $\underline{\theta}_{n}^{j *}+a$. Let $\underline{\Gamma}_{n}^{\#}, \bar{\Gamma}_{n}^{\#}, \underline{x}_{n}^{\#}, \bar{x}_{n}^{\#}$, $\underline{\theta}_{n+1}^{\#}, \bar{\theta}_{n+1}^{\#}$ be the functions obtained from $g_{n}^{\#}$ by (17)-(20) and (27)-(32). Proceeding as in the proof of Lemma $3, \underline{x}_{n}^{j \#} \leq \underline{x}_{n}^{j *}+a$, which implies $\underline{x}_{n, N D}^{j \#}-a \leq \underline{x}_{n}^{j \#}-a \leq \underline{x}_{n}^{j *}$. Then $\underline{x}_{n, N D}^{j \#}-a \in \underline{X}_{n}^{j *}=\left\{f \in \tilde{B}\left(R^{N-1}, \mathbb{R}\right) \mid f \leq \underline{x}_{n}^{j *}\right\}$, so from the definition of $\underline{x}_{n, N D}^{j *}$, $\underline{x}_{n, N D}^{j \#}-a \leq \underline{x}_{n, N D}^{j *}$, or equivalently, $\underline{x}_{n, N D}^{j \#} \leq \underline{x}_{n, N D}^{j *}+a$. Thus, arguing as in the proof of Lemma $3, \underline{\theta}_{n+1}^{j \#}\left(\theta^{-j}\right) \leq \underline{\theta}_{n+1}^{j *}\left(\theta^{-j}\right)+\lambda a$, where $\gamma=\sqrt{\beta} / \sqrt{2 \pi}$ and $\lambda \equiv \gamma /(1+\gamma) \in(0,1)$, and since the choice of $a \geq 0$ was arbitrary, discounting also holds.

Repeating the argument above for each component functions of $g_{n}^{*}$, with a minor modification for the $2 j$-th component functions, monotonicity and discounting holds for $\underline{\theta}_{n}^{j *}$ and $\bar{\theta}_{n}^{j *}, j=\{1,2, \ldots, N\}$. Thus, proceeding as in the Proof of Lemma $3, T^{\prime}$ is shown to be a contraction with the factor of contraction $\lambda$, so by the contraction mapping theorem, $T^{\prime}$ has a unique fixed point, denoted as $\left(\underline{\theta}^{*}, \bar{\theta}^{*}\right)$

\section{Proposition 3}

Given Proposition 2, it remains to establish the properties of the fixed point $\left(\underline{\theta}^{*}, \bar{\theta}^{*}\right)$, such as $\underline{\theta}^{*}=\bar{\theta}^{*} \equiv \theta^{*}$, and this is where one must resort to symmetry. Take $g_{n}^{*}=\left(\underline{\theta}_{n}^{*}, \bar{\theta}_{n}^{*}\right) \in$ $\tilde{B}\left(\mathbb{R}^{N}, \mathbb{R}^{2 N}\right)$ such that $\underline{\theta}_{n}^{*}=\bar{\theta}_{n}^{*} \equiv \theta_{n}^{*}=\left(\theta_{n}^{1 *}, \theta_{n}^{2 *}, \ldots, \theta_{n}^{N *}\right)$, where $\theta_{n}^{j *}$ is symmetric across $j$. From (17) and (18), $\underline{\Gamma}_{n}^{j *}=\bar{\Gamma}_{n}^{j *} \equiv \Gamma_{n}^{j *}$. Moreover, $\Gamma_{n}^{j *}$ is symmetric across $j$, and $\Gamma_{n}^{j *}$ and $\Gamma_{n}^{j *}-\Gamma_{n}^{k *}$ are continuous and decreasing in $x_{i}^{j}$, so (19) and (20) imply $\underline{x}_{n}^{j *}=\bar{x}_{n}^{j *} \equiv x_{n}^{j *}$, 
where $x_{n}^{j *}$ is symmetric across $j$.

Furthermore, $x_{n}^{j *}$ is nondecreasing, as shown below. Clearly $x_{n}^{j *}$ is nondecreasing when it equals $-\infty$, so suppose $x_{n}^{j *}$ is finite. Pick any $j, k \in\{1,2, \ldots, N\}, j \neq k$. Fix $x_{i}^{-j} \in \mathbb{R}^{N-1}$, and let $x_{n}^{j, k}\left(x_{i}^{-j}\right)$ be the value of $x_{i}^{j}$ such that

$$
\Gamma_{n}^{j *}\left(x_{i}\right)=\Gamma_{n}^{k *}\left(x_{i}\right) .
$$

Since $\Gamma_{n}^{j *}$ is symmetric across $j,(36)$ is satisfied for $x_{i}^{j}=x_{i}^{k}$, and since $\Gamma_{n}^{j *}\left(x_{i}\right)-\Gamma_{n}^{k *}\left(x_{i}\right)$ is decreasing in $x_{i}^{j}$, no other $x_{i}^{j}$ satisfies (36). Therefore, $x_{n}^{j, k}\left(x_{i}^{-j}\right)=x_{i}^{k}$, which is continuous and increasing in $x_{i}^{k}$, and is independent of other elements of $x_{i}^{-j}$. Now, with a slight abuse of notation, let $x_{n}^{j, 0}\left(x_{i}^{-j}\right)$ be the value of $x_{i}^{j}$ such that

$$
\Gamma_{n}^{j *}\left(x_{i}\right)=0 .
$$

Since $\Gamma_{n}^{j *}\left(x_{i}\right)$ is continuous, decreasing in $x_{i}^{j}$ and nondecreasing in $x_{i}^{-j}, x_{n}^{j, 0}\left(x_{i}^{-j}\right)$ is uniquely determined, and is continuous and nondecreasing in $x_{i}^{-j}$. Then, (19) (or equivalently, (20)) implies

$$
x_{n}^{j *}\left(x_{i}^{-j}\right)=\min \left\{x_{n}^{j, 0}\left(x_{i}^{-j}\right),\left(x_{n}^{j, k}\left(x_{i}^{-j}\right)\right)_{k \neq j}\right\}=\min \left\{x_{n}^{j, 0}\left(x_{i}^{-j}\right),\left(x_{i}^{k}\right)_{k \neq j}\right\} .
$$

So, $x_{n}^{j *}$ is continuous and nondecreasing in $x_{i}^{-j}$, and is increasing in $x_{i}^{k} \in x_{i}^{-j}$ in the region of $x_{i}^{-j} \in \mathbb{R}^{N-1}$ for which $x_{n}^{j *}\left(x_{i}^{-j}\right)=x_{n}^{j, k}\left(x_{i}^{-j}\right)=x_{i}^{k}$. But since $\underline{x}_{n}^{j *}=\bar{x}_{n}^{j *}=x_{n}^{j *}$ is nondecreasing, $\underline{x}_{n}^{j *}=\underline{x}_{n, N D}^{j *}=\bar{x}_{n}^{j *}=\bar{x}_{n, N D}^{j *}$. Thus, (31) and (32) imply $\underline{\theta}_{n+1}^{*}=\bar{\theta}_{n+1}^{*} \equiv$ $\theta_{n+1}^{*}=\left(\theta_{n+1}^{1 *}, \theta_{n+1}^{2 *}, \ldots, \theta_{n+1}^{N *}\right)$, where $\theta_{n+1}^{j *}$ is symmetric across $j, \theta_{n+1}^{j *} \in(0,1)$, and $\theta_{n+1}^{j *}$ is continuous and increasing.

Finally, let $\tilde{B}^{\prime}\left(\mathbb{R}^{N}, \mathbb{R}^{2 N}\right)$ denote the space of bounded, continuous, nondecreasing vector-valued functions $g: \mathbb{R}^{N} \rightarrow \mathbb{R}^{2 N}, g=\left(g^{1}, g^{2}, \ldots, g^{2 N}\right)$, where $g^{j}$ is symmetric across $j$ and $g^{j}=g^{2 j}$ for $j=\{1,2, \ldots, N\}$, equipped with the max-sup norm $\|g\| \equiv \max _{j=1}^{N}\left(\sup _{z \in \mathbb{R}}\left|g^{j}(z)\right|\right)$. Further, let $\tilde{B}^{\prime \prime}\left(\mathbb{R}^{N}, \mathbb{R}^{2 N}\right) \subseteq \tilde{B}^{\prime}\left(\mathbb{R}^{N}, \mathbb{R}^{2 N}\right)$ be the space in which 'nondecreasing' in the definition of $\tilde{B}^{\prime}\left(\mathbb{R}^{N}, \mathbb{R}^{2 N}\right)$ is replaced by 'increasing', and $g^{j} \in(0,1)$ for all $j$. Then, $\tilde{B}^{\prime}\left(\mathbb{R}^{N}, \mathbb{R}^{2 N}\right)$ is a closed subset of $\tilde{B}\left(\mathbb{R}^{N}, \mathbb{R}^{2 N}\right)$, and the argument above implies $T\left(\tilde{B}^{\prime}\left(\mathbb{R}^{N}, \mathbb{R}^{2 N}\right)\right) \subseteq \tilde{B}^{\prime \prime}\left(\mathbb{R}^{N}, \mathbb{R}^{2 N}\right) \subseteq \tilde{B}\left(\mathbb{R}^{N}, \mathbb{R}^{2 N}\right)$, so it follows that $g^{*} \in \tilde{B}^{\prime \prime}\left(\mathbb{R}^{N}, \mathbb{R}^{2 N}\right)$ (Stokey and Lucas (1989), Theorem 3.2, Corollary 1). Therefore, $\underline{\theta}^{*}=\bar{\theta}^{*} \equiv \theta^{*}=\left(\theta^{1 *}, \theta^{2 *}, \ldots, \theta^{N *}\right)$, where $\theta^{j *}$ is symmetric across $j, \theta^{j *} \in(0,1)$ and $\theta^{j *}$ is continuous and increasing. Then, at this fixed point, the expected payoff functions and the threshold signal functions must be such that $\underline{\Gamma}^{j *}=\bar{\Gamma}^{j *} \equiv \Gamma^{j *}$ and $\underline{x}^{j *}=\bar{x}^{j *} \equiv x^{j *}$, where $x^{j *}$ is continuous and nondecreasing. That $x^{j *}\left(x_{i}^{-j}\right) \leq \min _{k \neq j} x_{i}^{k}$ also follows since, by the argument above,

$$
x^{j *}\left(x_{i}^{-j}\right)=\min \left\{x^{j, 0}\left(x_{i}^{-j}\right),\left(x_{i}^{k}\right)_{k \neq j}\right\},
$$

where $x^{j, 0}\left(x_{i}^{-j}\right)$ is the value of $x_{i}^{j}$ such that $\Gamma^{j *}\left(x_{i}\right)=0$. 


\section{Proposition 4}

Let us first consider the $N=2$ case. Among the equations (17)-(22) that define the operator $T$, (17) and (18) are modified as follows. This time, conditional on receiving $x_{i}^{j}, \theta^{j}$ follows $N\left(\left(\alpha^{j} y^{j}+\beta^{j} x_{i}^{j}\right) /\left(\alpha^{j}+\beta^{j}\right), 1 /\left(\alpha^{j}+\beta^{j}\right)\right)$ such that

$\operatorname{Pr}\left(\theta^{j} \leq \theta^{j *} \mid x_{i}^{j}\right)=\Phi\left(\sqrt{\alpha^{j}+\beta^{j}}\left(\theta^{j *}-\frac{\alpha^{j} y^{j}+\beta^{j} x_{i}^{j}}{\alpha^{j}+\beta^{j}}\right)\right)$ for a given $\theta^{j *}$. Therefore

$$
\begin{aligned}
& \operatorname{Pr}\left(\theta^{j} \leq \underline{\theta}_{n}^{j *}\left(\theta^{-j}\right) \mid x_{i}^{j}, x_{i}^{-j}\right) \\
& =\int_{\epsilon_{i}^{-j} \in \mathbb{R}^{N-1}} \tilde{\phi}^{-j}\left(\epsilon_{i}^{-j}\right) \Phi\left(\sqrt{\alpha^{j}+\beta^{j}}\left(\underline{\theta}_{n}^{j *}\left(x_{i}^{-j}-\epsilon_{i}^{-j}\right)-\frac{\alpha^{j} y^{j}+\beta^{j} x_{i}^{j}}{\alpha^{j}+\beta^{j}}\right)\right) d \epsilon_{i}^{-j},
\end{aligned}
$$

which implies

$\underline{\Gamma}_{n}^{j *}\left(x_{i}\right)=\int_{\epsilon_{i}^{-j} \in \mathbb{R}^{N-1}} \tilde{\phi}^{-j}\left(\epsilon_{i}^{-j}\right) \Phi\left(\sqrt{\alpha^{j}+\beta^{j}}\left(\underline{\theta}_{n}^{j *}\left(x_{i}^{-j}-\epsilon_{i}^{-j}\right)-\frac{\alpha^{j} y^{j}+\beta^{j} x_{i}^{j}}{\alpha^{j}+\beta^{j}}\right)\right) d \epsilon_{i}^{-j}-c^{j}$,

and similarly

$\bar{\Gamma}_{n}^{j *}\left(x_{i}\right)=\int_{\epsilon_{i}^{-j} \in \mathbb{R}^{N-1}} \tilde{\phi}^{-j}\left(\epsilon_{i}^{-j}\right) \Phi\left(\sqrt{\alpha^{j}+\beta^{j}}\left(\bar{\theta}_{n}^{j *}\left(x_{i}^{-j}-\epsilon_{i}^{-j}\right)-\frac{\alpha^{j} y^{j}+\beta^{j} x_{i}^{j}}{\alpha^{j}+\beta^{j}}\right)\right) d \epsilon_{i}^{-j}-c^{j}$.

It is straightforward to check that $T: \tilde{B}\left(\mathbb{R}^{2}, \mathbb{R}^{4}\right) \rightarrow \tilde{B}\left(\mathbb{R}^{2}, \mathbb{R}^{4}\right)$ and that monotonicity holds as in the model with only private information. For discounting, take any $g_{n}^{*}=$ $\left(\underline{\theta}_{n}^{1 *}\left(\theta^{2}\right), \underline{\theta}_{n}^{2 *}\left(\theta^{1}\right), \bar{\theta}_{n}^{1 *}\left(\theta^{2}\right), \bar{\theta}_{n}^{2 *}\left(\theta^{1}\right)\right) \in \tilde{B}\left(\mathbb{R}^{2}, \mathbb{R}^{4}\right)$. Then fix $j \in\{1,2\}$, take any $a \geq 0$ and define $g_{n}^{\#} \in \tilde{B}\left(\mathbb{R}^{2}, \mathbb{R}^{4}\right)$ by replacing the $j$-th component of $g_{n}^{*}$, namely $\underline{\theta}_{n}^{j *}$, by $\underline{\theta}_{n}^{j *}+a$. Let us denote by $\underline{\Gamma}_{n}^{\#}, \bar{\Gamma}_{n}^{\#}, \underline{x}_{n}^{\#}, \bar{x}_{n}^{\#}, \underline{\theta}_{n+1}^{\#}$ and $\bar{\theta}_{n+1}^{\#}$ the functions obtained from $g_{n}^{\#}$ by (41), (42), and (19)-(22). Then, noting that $\underline{\Gamma}_{n}^{j \#}\left(x_{i}\right)$ and $\underline{\Gamma}_{n}^{j *}\left(x_{i}\right)$ are decreasing in $x_{i}^{j}$, whereas $\bar{\Gamma}_{n}^{k \#}\left(x_{i}\right)$ and $\bar{\Gamma}_{n}^{k *}\left(x_{i}\right)$ are nondecreasing in $x_{i}^{j}$ for $k \neq j, \underline{x}_{n}^{j \#} \leq \underline{x}_{n}^{j *}+\beta^{-1}(\alpha+\beta) a$ from (41), (42), and (19). Now, define $\underline{\theta}_{n+1}^{j \# \#}\left(\theta^{-j}\right)$ by

$$
\begin{aligned}
& \underline{\theta}_{n+1}^{j \# \#}\left(\theta^{-j}\right) \\
& =\int_{\epsilon_{i}^{-j} \in \mathbb{R}^{N-1}} \tilde{\phi}^{-j}\left(\epsilon_{i}^{-j}\right) \Phi\left(\sqrt{\beta^{j}}\left(\underline{x}_{n}^{j *}\left(\theta^{-j}+\epsilon_{i}^{-j}\right)+a\left(\alpha^{j}+\beta^{j}\right) / \beta^{j}-\underline{\theta}_{n+1}^{j \# \#}\left(\theta^{-j}\right)\right)\right) d \epsilon_{i}^{-j} .
\end{aligned}
$$

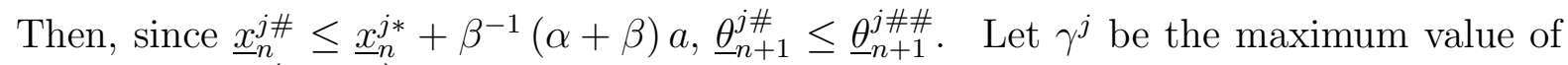
the slope of $\Phi\left(\sqrt{\beta^{j}} z\right)$ for $z \in \mathbb{R}$, that is, $\gamma^{j}=\sqrt{\beta^{j}} \phi(0)=\sqrt{\beta^{j}} / \sqrt{2 \pi}>0$. Then from 
(21) and (43),

$$
\begin{aligned}
& \underline{\theta}_{n+1}^{j \# \#}\left(\theta^{-j}\right)-\underline{\theta}_{n+1}^{j *}\left(\theta^{-j}\right) \\
& \leq \int_{\epsilon_{i}^{-j} \in \mathbb{R}^{N-1}} \tilde{\phi}^{-j}\left(\epsilon_{i}^{-j}\right) \gamma^{j}\left[a\left(\alpha^{j}+\beta^{j}\right) / \beta^{j}-\left(\underline{\theta}_{n+1}^{j \# \#}\left(\theta^{-j}\right)-\underline{\theta}_{n+1}^{j *}\left(\theta^{-j}\right)\right)\right] d \epsilon_{i}^{-j} \\
& \leq \gamma^{j}\left[a\left(\alpha^{j}+\beta^{j}\right) / \beta^{j}-\left(\underline{\theta}_{n+1}^{j \# \#}\left(\theta^{-j}\right)-\underline{\theta}_{n+1}^{j *}\left(\theta^{-j}\right)\right)\right] \\
& \leq a \lambda^{j}\left(\alpha^{j}+\beta^{j}\right) / \beta^{j}
\end{aligned}
$$

where $\lambda^{j} \equiv \gamma^{j} /\left(1+\gamma^{j}\right) \in(0,1)$, so $\underline{\theta}_{n+1}^{j \#}\left(\theta^{-j}\right) \leq \underline{\theta}_{n+1}^{j \# \#}\left(\theta^{-j}\right) \leq \underline{\theta}_{n+1}^{j *}\left(\theta^{-j}\right)+a \lambda^{j}\left(\alpha^{j}+\beta^{j}\right) / \beta^{j}$. But since $\alpha^{j}<\sqrt{\beta^{j}} \sqrt{2 \pi}$ by assumption,

$$
\lambda^{j}\left(\alpha^{j}+\beta^{j}\right) / \beta^{j}=\frac{\sqrt{\beta^{j}} / \sqrt{2 \pi}}{1+\sqrt{\beta^{j}} / \sqrt{2 \pi}} \frac{\alpha^{j}+\beta^{j}}{\beta^{j}}<\frac{\sqrt{\beta^{j}}}{\sqrt{2 \pi}+\sqrt{\beta^{j}}} \frac{\sqrt{2 \pi}+\sqrt{\beta^{j}}}{\sqrt{\beta^{j}}}=1,
$$

and since the choice of $a \geq 0$ was arbitrary, discounting also holds. The rest of the proof follows that of Proposition 1.

For the symmetric $N>2$ case, among the equations (17)-(20) and (27)-(32) that define the operator $T^{\prime},(17)$ and (18) are modified as (41) and (42), and $c^{j}=c, \beta^{j}=\beta$ in all equations. It is again straightforward to check that $T^{\prime}: \tilde{B}\left(\mathbb{R}^{N}, \mathbb{R}^{2 N}\right) \rightarrow \tilde{B}\left(\mathbb{R}^{N}, \mathbb{R}^{2 N}\right)$ and that monotonicity holds, and arguing as in the $N=2$ case above, discounting follows given $\alpha / \sqrt{\beta}<\sqrt{2 \pi}$. The rest of the proof follows that of Proposition 3.

\section{Proposition 5}

The argument requires only minor modifications to that for Propositions 1-3. When signals $\epsilon_{i}=\left(\epsilon_{i}^{1}, \epsilon_{i}^{2}, \ldots, \epsilon_{i}^{N}\right)=\left(\epsilon_{i}^{j}, \epsilon_{i}^{-j}\right)$ are drawn from a joint pdf $\varphi$, one can write, for example,

$$
\begin{aligned}
\operatorname{Pr}\left(\theta^{j} \leq \underline{\theta}_{n}^{j *}\left(\theta^{-j}\right) \mid x_{i}^{j}, x_{i}^{-j}\right) & =\operatorname{Pr}\left(x_{i}^{j}-\epsilon_{i}^{j} \leq \underline{\theta}_{n}^{j *}\left(x_{i}^{-j}-\epsilon_{i}^{-j}\right)\right) \\
& =\int_{\epsilon_{i}^{-j} \in \mathbb{R}^{N-1}} \int_{x_{i}^{j}-\underline{\theta}_{n}^{j *}\left(x_{i}^{-j}-\epsilon_{i}^{-j}\right)}^{\infty} \varphi\left(\epsilon_{i}\right) d \epsilon_{i}^{j} d \epsilon_{i}^{-j} .
\end{aligned}
$$


Thus, the equations (17)-(22) that define the operator $T$ are rewritten as

$$
\begin{aligned}
\underline{\Gamma}_{n}^{j *}\left(x_{i}\right) & =\int_{\epsilon_{i}^{-j} \in \mathbb{R}^{N-1}} \int_{x_{i}^{j}-\underline{\theta}_{n}^{j *}\left(x_{i}^{-j}-\epsilon_{i}^{-j}\right)}^{\infty} \varphi\left(\epsilon_{i}\right) d \epsilon_{i}^{j} d \epsilon_{i}^{-j}-c^{j}, \\
\bar{\Gamma}_{n}^{j *}\left(x_{i}\right) & =\int_{\epsilon_{i}^{-j} \in \mathbb{R}^{N-1}} \int_{x_{i}^{j}-\bar{\theta}_{n}^{j *}\left(x_{i}^{-j}-\epsilon_{i}^{-j}\right)} \varphi\left(\epsilon_{i}\right) d \epsilon_{i}^{j} d \epsilon_{i}^{-j}-c^{j}, \\
\underline{x}_{n}^{j *}\left(x_{i}^{-j}\right) & =\inf \left\{x_{i}^{j} \mid \underline{\Gamma}_{n}^{j *}\left(x_{i}\right) \leq \max \left\{0,\left(\bar{\Gamma}_{n}^{k *}\left(x_{i}\right)\right)_{k \neq j}\right\}\right\} \text { if inf exists, } \\
& =-\infty \text { otherwise, } \\
\bar{x}_{n}^{j *}\left(x_{i}^{-j}\right) & =\sup \left\{x_{i}^{j} \mid \bar{\Gamma}_{n}^{j *}\left(x_{i}\right) \geq \max \left\{0,\left(\underline{\Gamma}_{n}^{k *}\left(x_{i}\right)\right)_{k \neq j}\right\}\right\} \text { if sup exists, } \\
& =-\infty \text { otherwise, } \\
\underline{\theta}_{n+1}^{j *}\left(\theta^{-j}\right) & =\int_{\epsilon_{i}^{-j} \in \mathbb{R}^{N-1}} \int_{-\infty} \underline{x}_{n}^{j *}\left(\theta^{-j}+\epsilon_{i}^{-j}\right)-\underline{\theta}_{n+1}^{j *}\left(\theta^{-j}\right) \\
\bar{\theta}_{n+1}^{j *}\left(\theta^{-j}\right) & =\int_{\epsilon_{i}^{-j} \in \mathbb{R}^{N-1}} \int_{-\infty}^{\bar{x}_{n}^{j *}\left(\theta^{-j}+\epsilon_{i}^{-j}\right)-\bar{\theta}_{n+1}^{j *}\left(\theta^{-j}\right)} \varphi\left(\epsilon_{i}\right) d \epsilon_{i}^{j} d \epsilon_{i}^{-j} .
\end{aligned}
$$

Suppose $N=2$. Lemma 1 clearly does not hinge on these equations. Lemma 2 follows from the same argument as when noises are independent. Regarding Lemma 3, monotonicity can be shown as before. For discounting, proceed as before to obtain

$$
\underline{\theta}_{n+1}^{j \# \#}\left(\theta^{-j}\right)=\int_{\epsilon_{i}^{-j} \in \mathbb{R}^{N-1}} \int_{-\infty}^{\underline{x}_{n}^{j *}\left(\theta^{-j}+\epsilon_{i}^{-j}\right)+a-\underline{\theta}_{n+1}^{j \# \#}\left(\theta^{-j}\right)} \varphi\left(\epsilon_{i}\right) d \epsilon_{i}^{j} d \epsilon_{i}^{-j} .
$$

Let $\varphi^{-j}\left(\epsilon_{i}^{-j}\right)$ denote the marginal pdf of $\epsilon_{i}^{-j}, \varphi^{j}\left(\epsilon_{i}^{j} \mid \epsilon_{i}^{-j}\right)$ be the conditional pdf of $\epsilon_{i}^{j}$ given $\epsilon_{i}^{-j}$, and define $\gamma^{j} \equiv \sup _{\epsilon_{i}} \varphi^{j}\left(\epsilon_{i}^{j} \mid \epsilon_{i}^{-j}\right)$. Then, from (50) and (52),

$$
\begin{aligned}
& \underline{\theta}_{n+1}^{j \# \#}\left(\theta^{-j}\right)-\underline{\theta}_{n+1}^{j *}\left(\theta^{-j}\right) \\
& =\int_{\epsilon_{i}^{-j} \in \mathbb{R}^{N-1}} \varphi^{-j}\left(\epsilon_{i}^{-j}\right) \int_{\underline{x}_{n}^{j *}\left(\theta^{-j}+\epsilon_{i}^{-j}\right)-\underline{\theta}_{n+1}^{j *}\left(\theta^{-j}\right)}^{\underline{x}_{n}^{j *}\left(\theta^{-j}+\epsilon_{i}^{-j}\right)+a-\underline{\theta}_{n+1}^{j \# \#}\left(\theta^{-j}\right)} \varphi^{j}\left(\epsilon_{i}^{j} \mid \epsilon_{i}^{-j}\right) d \epsilon_{i}^{j} d \epsilon_{i}^{-j} \\
& \leq \int_{\epsilon_{i}^{-j} \in \mathbb{R}^{N-1}} \varphi^{-j}\left(\epsilon_{i}^{-j}\right) \gamma^{j}\left(a-\left(\underline{\theta}_{n+1}^{j \# \#}\left(\theta^{-j}\right)-\underline{\theta}_{n+1}^{j *}\left(\theta^{-j}\right)\right)\right) d \epsilon_{i}^{-j} \\
& \leq \gamma^{j}\left(a-\left(\underline{\theta}_{n+1}^{j \# \#}\left(\theta^{-j}\right)-\underline{\theta}_{n+1}^{j *}\left(\theta^{-j}\right)\right)\right) \\
& \leq \lambda^{j} a
\end{aligned}
$$

where $\lambda^{j} \equiv \gamma^{j} /\left(1+\gamma^{j}\right) \in(0,1)$, which establishes discounting. The remaining part of the proof follows that of Proposition 1.

For the symmetric $N>2$ case, rewrite the equations that define the operator $T^{\prime}$, (17)-(20) and (27)-(32), using $\varphi$. Then discounting can be shown as for the $N=2$ case above, and the rest of the proof follows that of Propositions 2 and 3. 


\section{Appendix B: Computational Procedure}

For $N=2$, the operator $T$ defined by (17)-(22) satisfies the requirement of the contraction mapping theorem, so the equilibrium functions can be obtained by iterating on these equations from any $g_{0}^{*}(\theta)=\left(\underline{\theta}_{0}^{1 *}\left(\theta^{2}\right), \underline{\theta}_{0}^{2 *}\left(\theta^{1}\right), \bar{\theta}_{0}^{1 *}\left(\theta^{2}\right), \bar{\theta}_{0}^{2 *}\left(\theta^{1}\right)\right) \in \tilde{B}\left(\mathbb{R}^{2}, \mathbb{R}^{4}\right)$. But then, one can choose $g_{0}^{*}$ such that $\underline{\theta}_{0}^{j *}=\bar{\theta}_{0}^{j *} \equiv \theta_{0}^{j *}$ for $j=\{1,2\}$, in which case $g_{1}^{*}(\theta)=\left(\underline{\theta}_{1}^{1 *}\left(\theta^{2}\right), \underline{\theta}_{1}^{2 *}\left(\theta^{1}\right), \bar{\theta}_{1}^{1 *}\left(\theta^{2}\right), \bar{\theta}_{1}^{2 *}\left(\theta^{1}\right)\right)$ is such that $\underline{\theta}_{1}^{j *}=\bar{\theta}_{1}^{j *} \equiv \theta_{1}^{j *}$ for $j=\{1,2\}$, as argued in the proof of Proposition 1. Thus, one can iterate instead on the equations,

$$
\begin{aligned}
\Gamma_{n}^{j *}\left(x_{i}\right) & =\int_{\epsilon_{i}^{-j} \in \mathbb{R}^{N-1}} \tilde{\phi}^{-j}\left(\epsilon_{i}^{-j}\right) \Phi\left(\sqrt{\beta^{j}}\left(\theta_{n}^{j *}\left(x_{i}^{-j}-\epsilon_{i}^{-j}\right)-x_{i}^{j}\right)\right) d \epsilon_{i}^{-j}-c^{j}, \\
x_{n}^{j *}\left(x_{i}^{-j}\right) & =\left\{x_{i}^{j} \mid \Gamma_{n}^{j *}\left(x_{i}\right)=\max \left\{0,\left(\Gamma_{n}^{k *}\left(x_{i}\right)\right)_{k \neq j}\right\}\right\} \text { if such } x_{i}^{j} \text { exists, } \\
& =-\infty \text { otherwise, } \\
\theta_{n+1}^{j *}\left(\theta^{-j}\right) & =\int_{\epsilon_{i}^{-j} \in \mathbb{R}^{N-1}} \tilde{\phi}^{-j}\left(\epsilon_{i}^{-j}\right) \Phi\left(\sqrt{\beta^{j}}\left(x_{n}^{j *}\left(\theta^{-j}+\epsilon_{i}^{-j}\right)-\theta_{n+1}^{j *}\left(\theta^{-j}\right)\right)\right) d \epsilon_{i}^{-j} .
\end{aligned}
$$

for $j, k \in\{1,2\}, j \neq k$, starting from any $\left(\theta_{0}^{1 *}, \theta_{0}^{2 *}\right) \in \tilde{B}\left(\mathbb{R}^{2}, \mathbb{R}^{2}\right) .^{22}$

For the symmetric case, including $N=2$, the operator $T^{\prime}$ defined by (17)-(20) and (27)-(32) satisfies the requirement of the contraction mapping theorem, so the equilibrium functions can be obtained by iterating on these equations from any $g_{0}^{*}(\theta) \in \tilde{B}\left(\mathbb{R}^{N}, \mathbb{R}^{2 N}\right)$. However, there are a number of possible simplifications. As discussed in the proof of Proposition 3, if $g_{0}^{*}(\theta)$ is such that $\underline{\theta}_{0}^{j *}=\bar{\theta}_{0}^{j *} \equiv \theta_{0}^{j *}$ for $j=\{1,2, \ldots, N\}$, where $\theta_{0}^{j *}$ is symmetric across $j$, then $\underline{x}_{0}^{j *}=\bar{x}_{0}^{j *}=\underline{x}_{0, N D}^{j *}=\bar{x}_{0, N D}^{j *} \equiv x_{0}^{j *}$, where $x_{0}^{j *}$ is symmetric across $j$; this implies $\underline{\theta}_{1}^{j *}=\bar{\theta}_{1}^{j *} \equiv \theta_{1}^{j *}$, where $\theta_{1}^{j *}$ is symmetric across $j$. So, starting from such $g_{0}^{*}$, the operators $T^{\prime}$ and $T$ are equivalent. Thus, one can iterate on (53)-(55) for a single $j$, and set all $\Gamma_{n}^{k *}$ to be symmetric to $\Gamma_{n}^{j *}$. Moreover, for $\left(\theta_{n}^{1 *}, \theta_{n}^{2 *}, \ldots, \theta_{n}^{N *}\right) \in \tilde{B}\left(\mathbb{R}^{N}, \mathbb{R}^{N}\right)$ such that $\theta_{n}^{j *}$ is symmetric across $j, x_{n}^{j *}$ can be expressed as (38). In summary, one can choose any $\theta_{0}^{j *} \in \tilde{B}\left(\mathbb{R}^{N-1}, \mathbb{R}\right)$ and iterate to convergence on

$$
\begin{aligned}
\Gamma_{n}^{j *}\left(x_{i}\right) & =\int_{\epsilon_{i}^{-j} \in \mathbb{R}^{N-1}} \tilde{\phi}^{-j}\left(\epsilon_{i}^{-j}\right) \Phi\left(\sqrt{\beta}\left(\theta_{n}^{j *}\left(x_{i}^{-j}-\epsilon_{i}^{-j}\right)-x_{i}^{j}\right)\right) d \epsilon_{i}^{-j}-c, \\
x_{n}^{j *}\left(x_{i}^{-j}\right) & =\min \left\{x_{n}^{j, 0}\left(x_{i}^{-j}\right),\left(x_{i}^{k}\right)_{k \neq j}\right\}, \Gamma_{n}^{j *}\left(x_{n}^{j, 0}\left(x_{i}^{-j}\right), x_{i}^{-j}\right)=0, \\
\theta_{n+1}^{j *}\left(\theta^{-j}\right) & =\int_{\epsilon_{i}^{-j} \in \mathbb{R}^{N-1}} \tilde{\phi}^{-j}\left(\epsilon_{i}^{-j}\right) \Phi\left(\sqrt{\beta}\left(x_{n}^{j *}\left(\theta^{-j}+\epsilon_{i}^{-j}\right)-\theta_{n+1}^{j *}\left(\theta^{-j}\right)\right)\right) d \epsilon_{i}^{-j},
\end{aligned}
$$

for a single $j$, and resort to symmetry to obtain functions for other $j$.

\footnotetext{
${ }^{22}$ Since $\Gamma_{n}^{j *}, \Gamma_{n}^{j *}-\Gamma_{n}^{k *}$ decrease continuously in $x_{i}^{j}$, the set $\left\{x_{i}^{j} \mid \Gamma_{n}^{j *}\left(x_{i}\right)=\max \left\{0,\left(\Gamma_{n}^{k *}\left(x_{i}\right)\right)_{k \neq j}\right\}\right\}$ is empty, or has a unique element that equals both $\inf \left\{x_{i}^{j} \mid \Gamma_{n}^{j *}\left(x_{i}\right) \leq \max \left\{0,\left(\Gamma_{n}^{k *}\left(x_{i}\right)\right)_{k \neq j}\right\}\right\}$ and $\sup \left\{x_{i}^{j} \mid \Gamma_{n}^{j *}\left(x_{i}\right) \geq \max \left\{0,\left(\Gamma_{n}^{k *}\left(x_{i}\right)\right)_{k \neq j}\right\}\right\}$. So, (19) and (20) simplify as (54).
} 


\section{References}

Angeletos, G., C. Hellwig, and A. Pavan (2007): "Dynamic Global Games of Regime Change: Learning, Multiplicity, and the Timing of Attacks," Econometrica, $75(3), 711-756$.

Angeletos, G., And I. Werning (2006): "Crises and Prices: Information Aggregation, Multiplicity, and Volatility," American Economic Review, 96(5), 1720-1736.

Atkeson, A. (2000): "Discussion of Morris and Shin's 'Rethinking Multiple Equilibria in Macroeconomic Modelling,'" in NBER Macroeconomics Annual, ed. by B. Bernanke, and K. Rogoff, pp. 162-171. Cambridge, MA: MIT Press.

Dasgupta, A. (2007): "Coordination and Delay in Global Games," Journal of Economic Theory, 134(1), 195-225.

Edelstein, M. (1962): "On Fixed and Periodic Points under Contractive Mappings," Journal of the London Mathematical Society, 1(1), 74-79.

Frankel, D.-M., S. Morris, and A. Pauzner (2003): "Equilibrium Selection in Global Games with Strategic Complementarities," Journal of Economic Theory, 108(1), $1-44$.

Heinemann, F., And G. Illing (2002): "Speculative Attacks: Unique Equilibrium and Transparency," Journal of International Economics, 58(2), 429-50.

Hellwig, C. (2002): "Public Information, Private Information, and the Multiplicity of Equilibria in Coordination Games," Journal of Economic Theory, 107(2), 191-222.

Hellwig, C., A. Mukherji, And A. Tsyvinski (2006): "Self-fulfilling Currency Crises: The Role of Interest Rates," American Economic Review, 96(5), 1769-1787.

Levin, J. (2001): "A Note on Global Equilibrium Selection in Overlapping Generations Games," mimeo, Stanford University.

Mason, R., And A. Valentinyi (2007): "The Existence and Uniqueness of Monotone Pure Strategy Equilibrium in Bayesian Games," University of Southampton Discussion Paper Series in Economics and Econometrics 0710.

Mathevet, L. (2010): "A Contraction Principle for Finite Global Games," Economic Theory, 42(3), 539-563. 
McAdams, D. (2003): "Isotone Equilibrium in Games of Incomplete Information," Econometrica, 71(4), 1191-1214.

Milgrom, P., and J. Roberts (1990): "Rationalizability, Learning, and Equilibrium in Games with Strategic Complementarities," Econometrica, 58(6), 1255-1277.

Morris, S., And H.-S. Shin (1998): "Unique Equilibrium in a Model of Self-Fulfilling Currency Attacks," American Economic Review, 88(3), 587-97.

(2002): "Global Games: Theory and Applications," in Advances in Economics and Econometrics, the Eighth World Congress, ed. by M. Dewatripoint, L.-P. Hansen, and S. Turnovsky. Cambridge: Cambridge Univesity Press.

Obstfeld, M. (1996): "Models of Currency Crises with Self-Fulfilling Features," European Economic Review, 40(3-5), 1037-1047.

Oury, M. (2005): "Multidimensional Global Games," mimeo, HEC School of Management.

Steiner, J. (2007): "Coordination of Mobile Labor," Journal of Economic Theory, $139(1), 25-46$.

Stokey, N., and R. Lucas (1989): Recursive Methods in Economic Dynamics. Cambridge, MA: Harvard University Press.

Vives, X. (1990): "Nash Equilibrium with Strategic Complementarities," Journal of Mathematical Economics, 19(3), 305-321. 


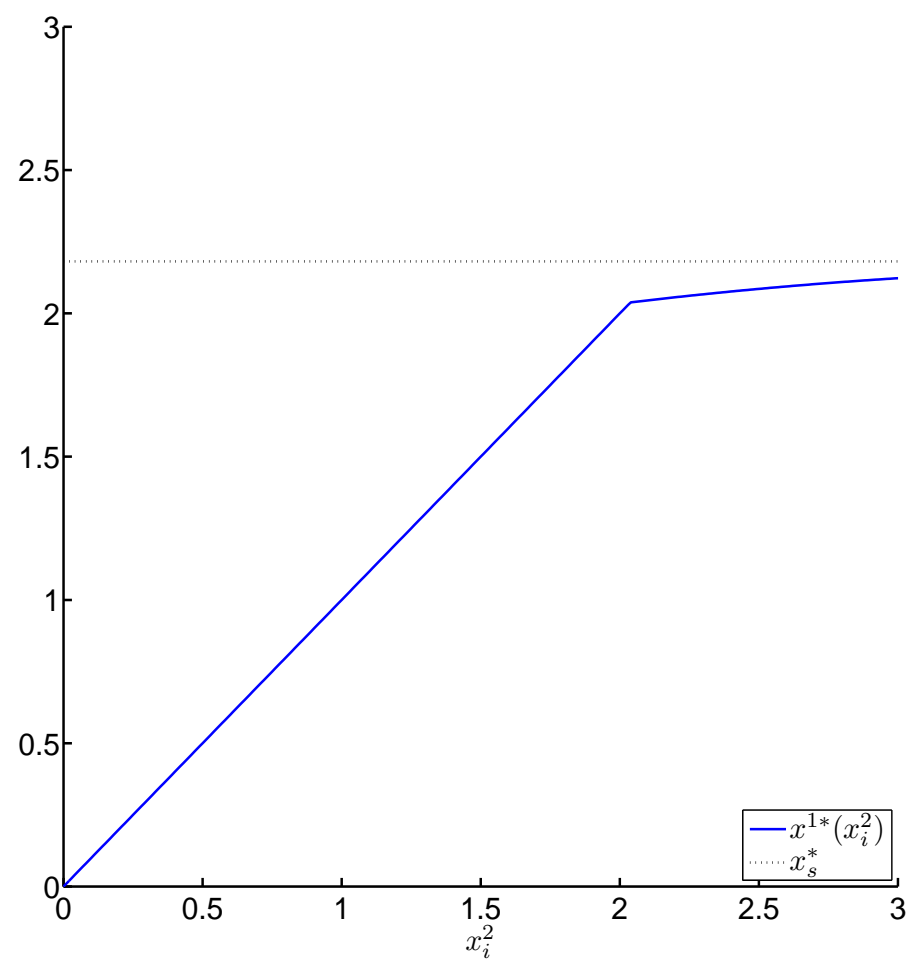

Figure 1: Threshold signal function (symmetric, $\beta=1$ ).

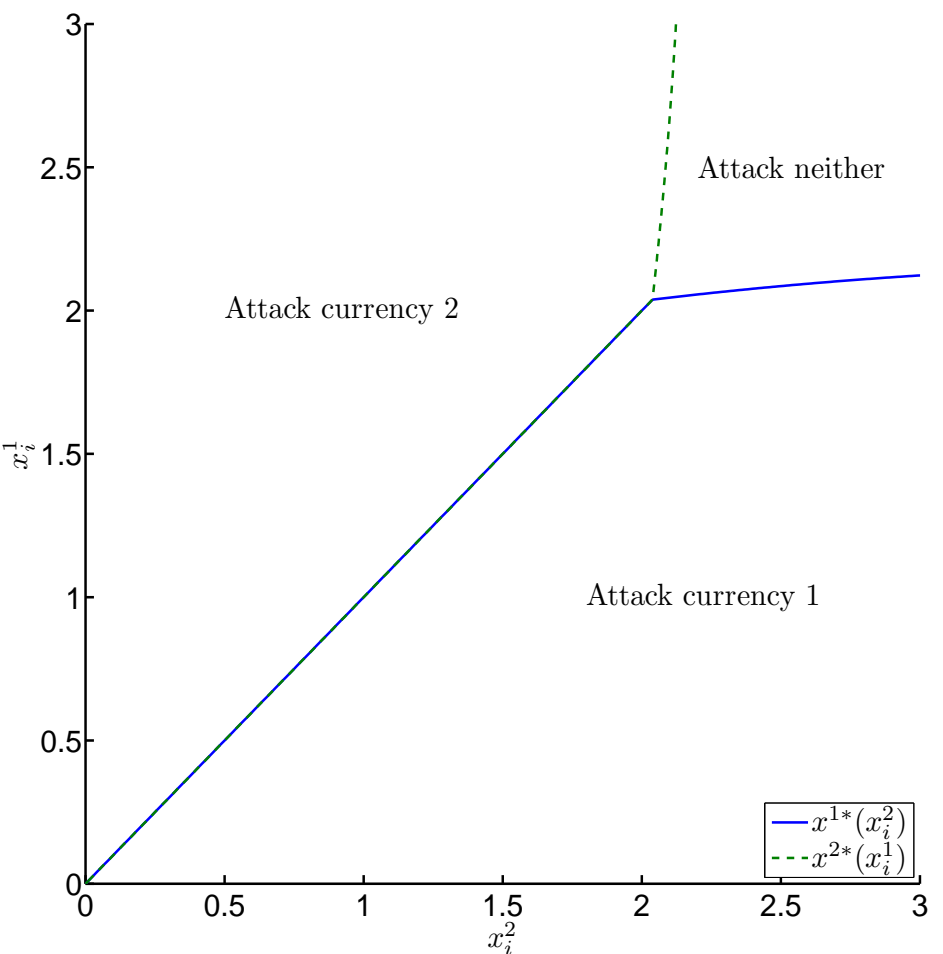

Figure 2: Attacking decisions (symmetric, $\beta=1$ ). 


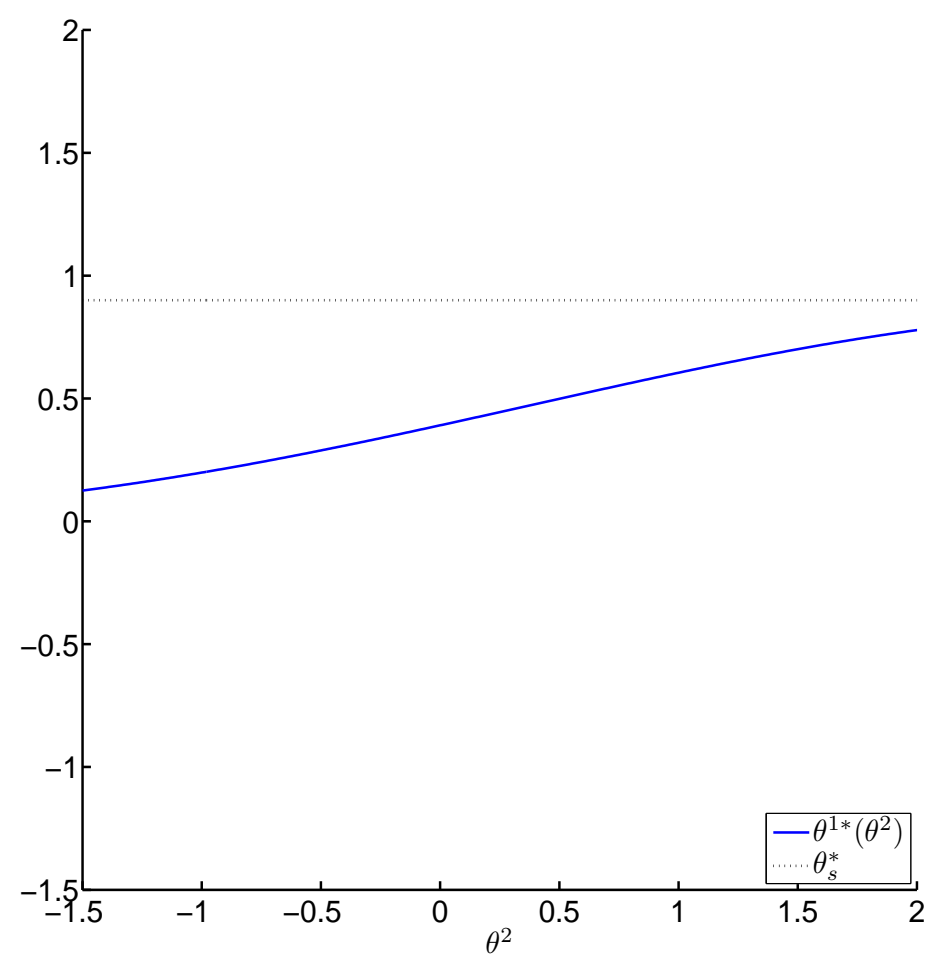

Figure 3: Threshold fundamentals function (symmetric, $\beta=1$ ).

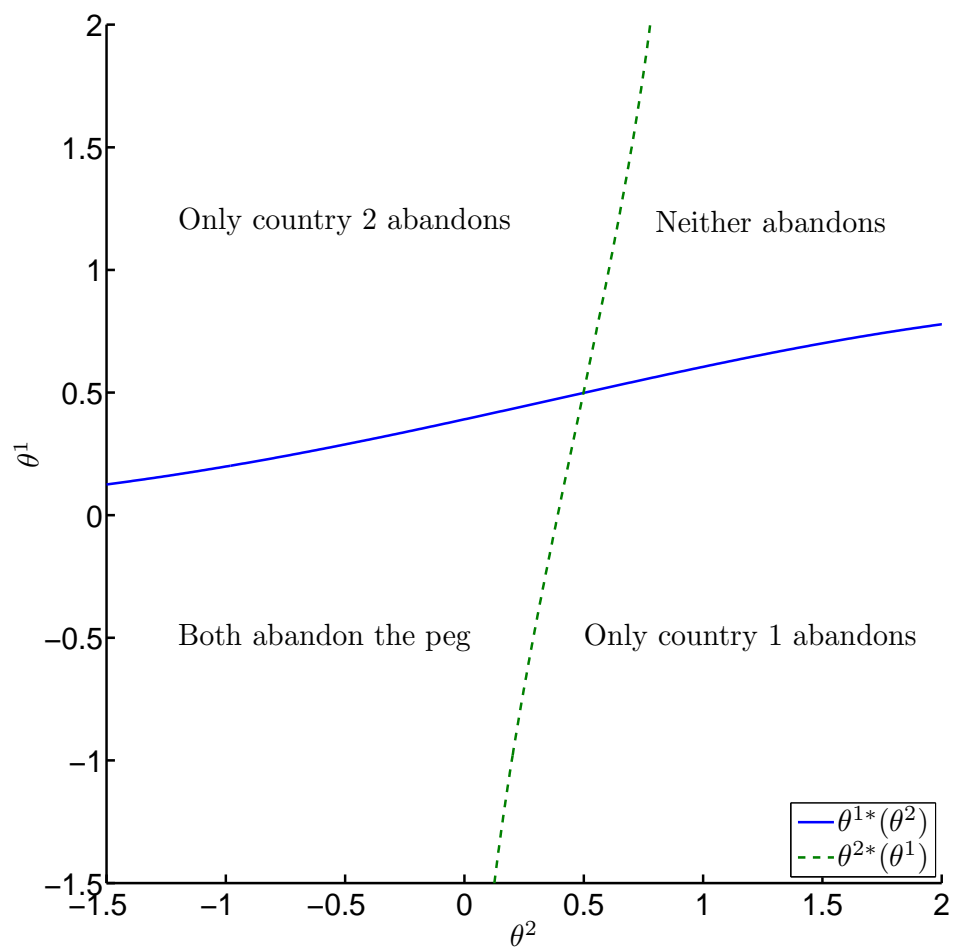

Figure 4: Outcomes of attack (symmetric, $\beta=1$ ). 


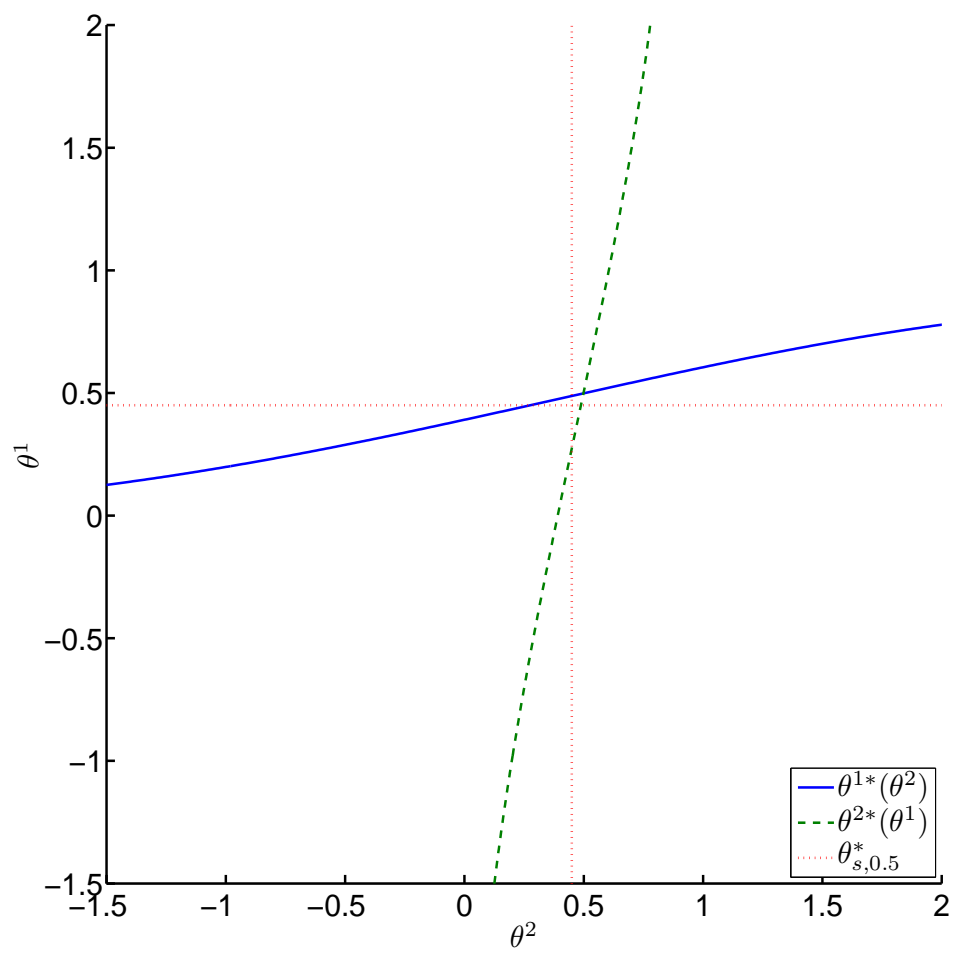

Figure 5: $\theta^{1 *}\left(\theta^{2}\right)$ and $\theta^{2 *}\left(\theta^{1}\right)$ compared with $\theta_{s, 0.5}^{*}($ symmetric, $\beta=1)$.

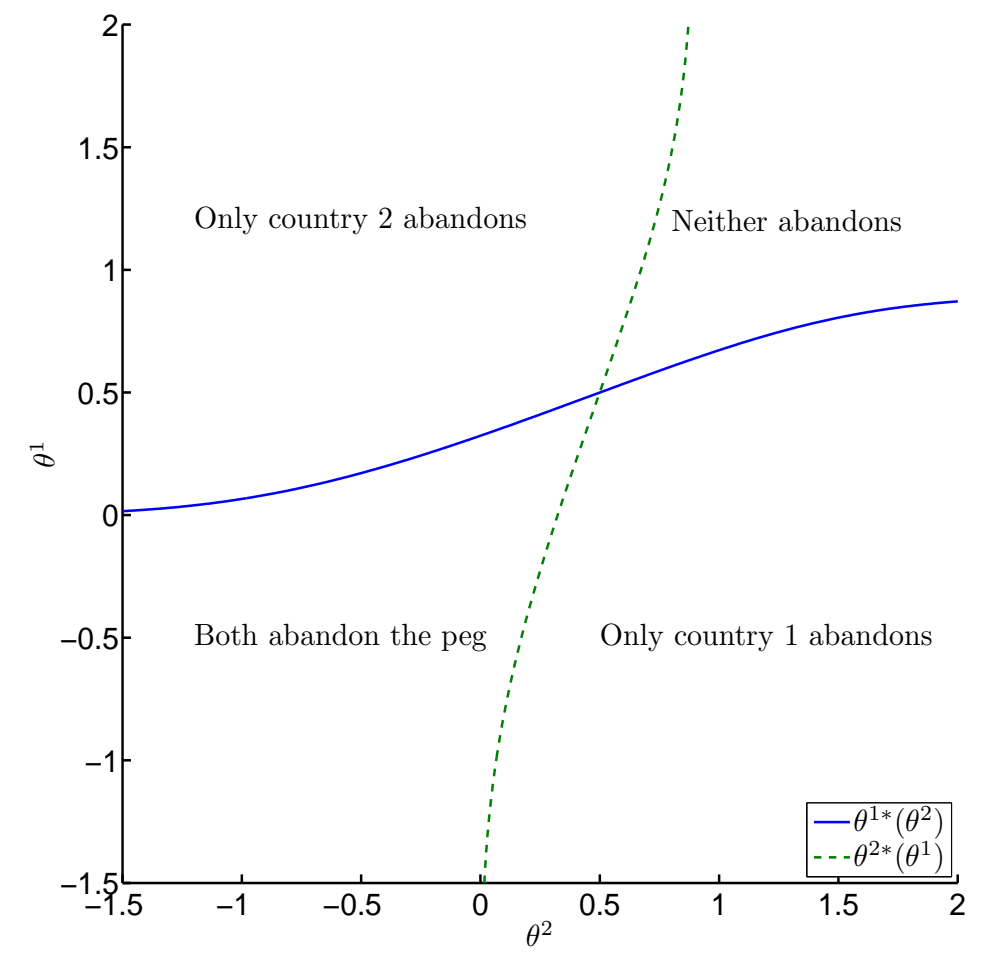

Figure 6: Outcomes of attack (symmetric, $\beta=4$ ). 


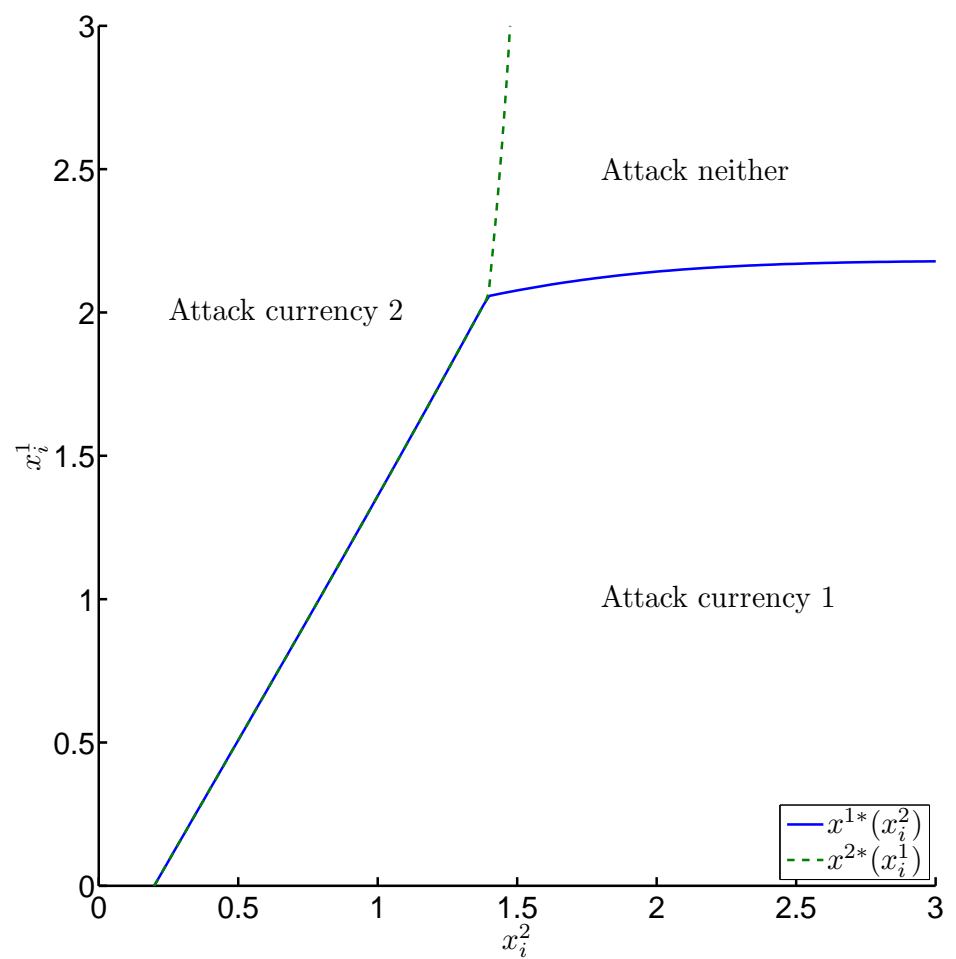

Figure 7: Attacking decisions $\left(\beta^{1}=1, \beta^{2}=4\right)$.

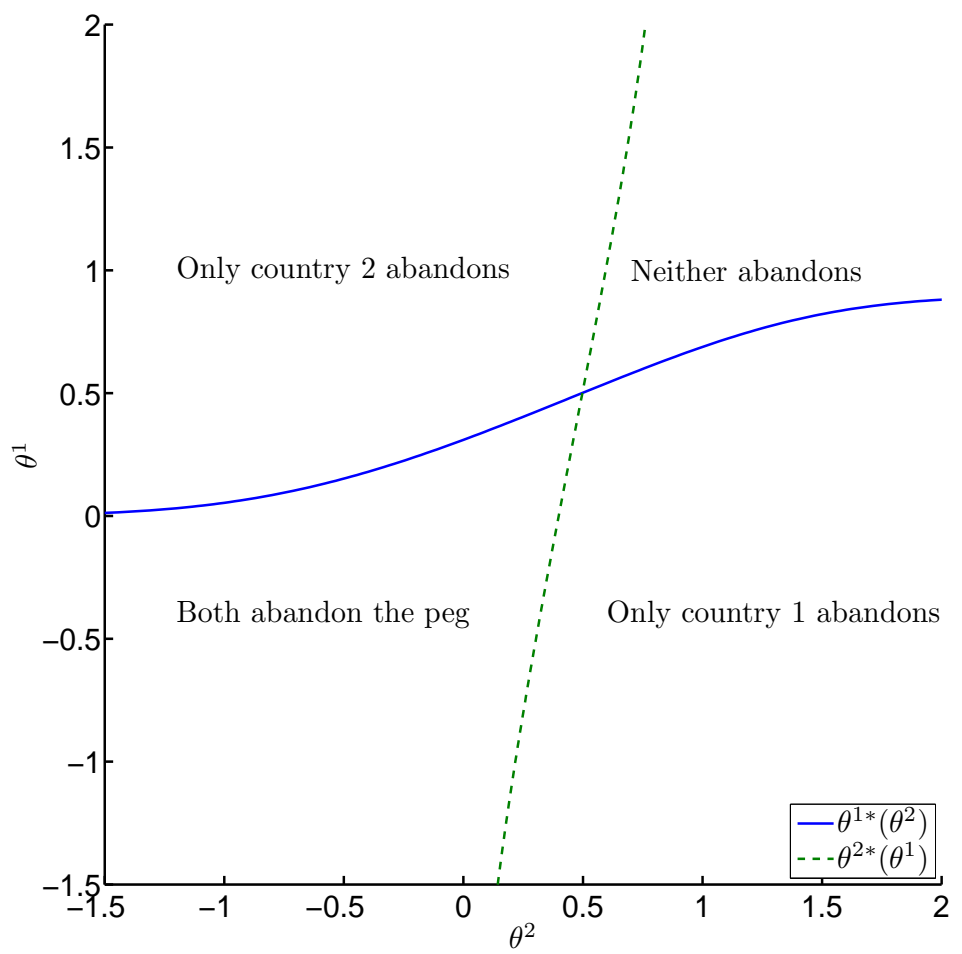

Figure 8: Outcomes of attack $\left(\beta^{1}=4, \beta^{2}=1\right)$. 


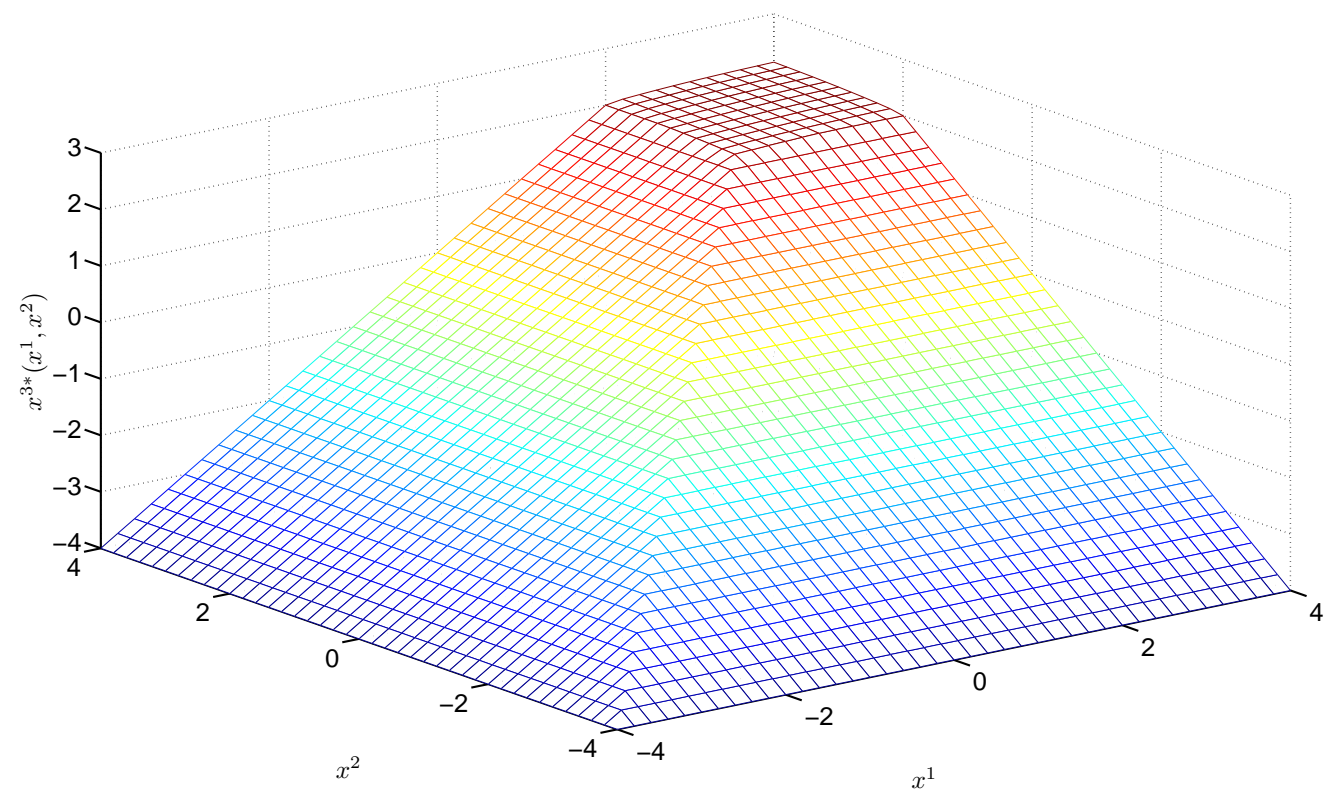

Figure 9: Threshold signal function $(N=3)$.

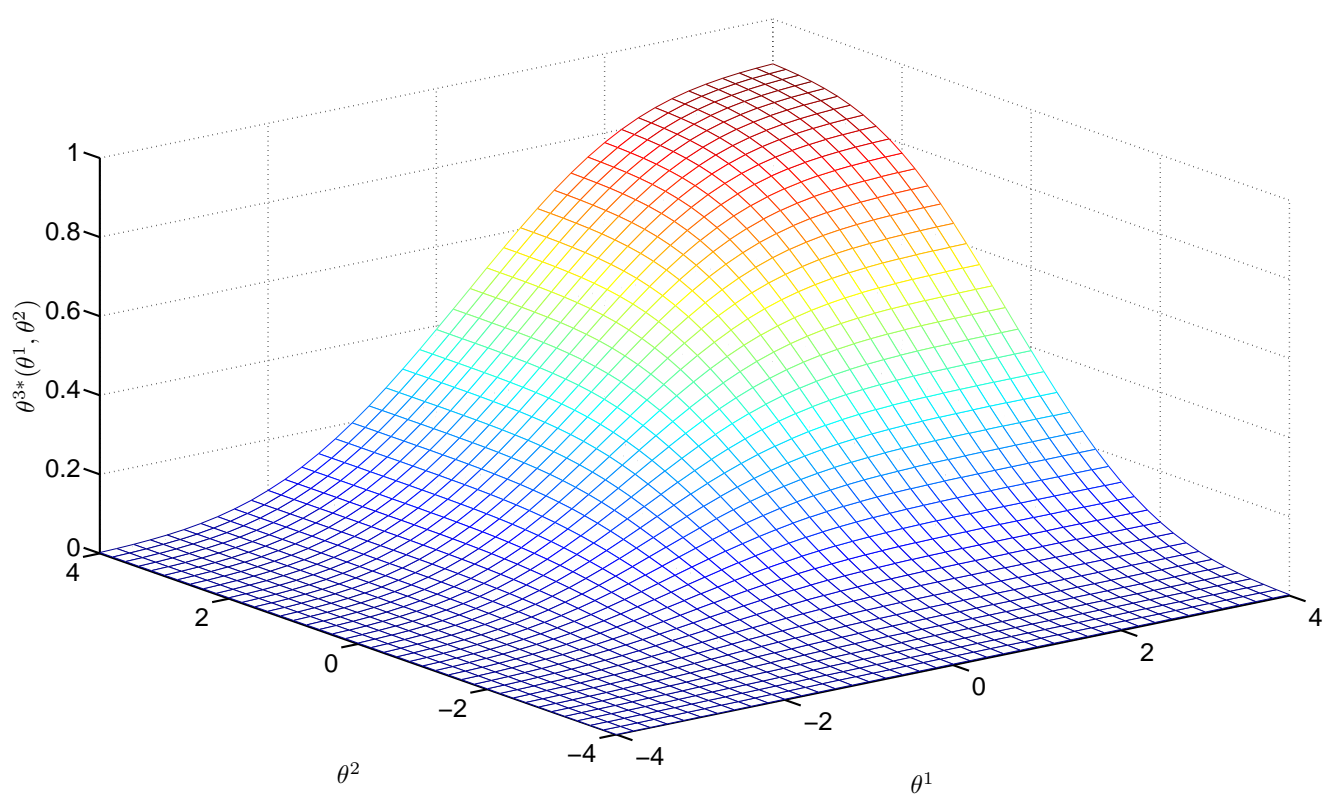

Figure 10: Threshold fundamentals function $(N=3)$. 